Pacific Journal of Mathematics

CHARACTER VALUE ESTIMATES FOR GROUPS OF LIE TYPE 


\title{
CHARACTER VALUE ESTIMATES FOR GROUPS OF LIE TYPE
}

\author{
DAVID GLUCK
}

\begin{abstract}
Let $G$ be a group of Lie type over the field of $q$ elements. Let $\chi$ be a nonlinear irreducible character of $G$ and $x$ a noncentral element of $G$. Examination of character tables suggests that $|\chi(x) / \chi(1)| \leq$ $C / q$, where $C$ is a universal constant independent of $\chi, x$, and $G$. This order of magnitude is attained when, for example, $\chi$ is the doubly transitive permutation character of $\mathrm{GL}(n, q)$ and $x$ centralizes a hyperplane of $\operatorname{PG}(n-1, q) ;|\chi(x) / \chi(1)|$ then approaches $1 / q$ as $n \rightarrow \infty$. In this paper, we establish a bound of the above type when $x$ is a semisimple element which has prime order modulo $Z(G)$. However, we must exclude certain groups $G$ in characteristic 2 and 3. The most serious exclusions are the groups of type $C_{n}$ in characteristic 2. Our proof, which is summarized below, does not use Deligne-Lusztig theory.
\end{abstract}

We first consider the case that $x$ is contained in no proper parabolic subgroup of $G$. By character orthogonality, $|\chi(x)| \leq\left|C_{G}(x)\right|^{1 / 2}$. Since $C_{G}(x)$ is essentially a torus, the lower bounds for $\chi(1)$ in [16] yield the desired upper bound for $|\chi(x) / \chi(1)|$.

We may then assume that $x$ is contained in a Levi complement $L_{J}$ of a suitable standard maximal parabolic $P_{J}$. We write $\chi_{P_{J}}=$ $\chi_{1}+\chi_{2}+\chi_{3}+\chi_{4}$, where the irreducible constituents of $\chi_{1}$ are linear characters of $P_{J}$, the irreducible constituents of $\chi_{2}$ are nonlinear but have $U_{J}$ in their kernels, the irreducible constituents of $\chi_{3}$ lie over nonprincipal $L_{J}^{\prime}$-invariant irreducible characters of $U_{J}$, and the irreducible constituents of $\chi_{4}$ lie over irreducible characters of $U_{J}$ which are not $L_{J}^{\prime}$-invariant.

We show that $\left|\chi_{1}(x)\right|$ is absolutely bounded by finding absolute upper bounds both for the multiplicities of the linear constituents of $\chi_{P_{J}}$ and for the number of distinct linear constituents of $\chi_{P_{J}}$. We essentially get the best possible absolute upper bound for the multiplicities. The theory developed in [13] and [17] is used to bound these multiplicities in terms of corresponding multiplicities in the Weyl group $W$ of $G$. We obtain only a crude absolute upper bound for the number of distinct linear constituents of $\chi_{P_{J}}$. Our bound involves the indices of certain large reflection subgroups of $W$ in their normalizers. 
Since $L_{J}$ is a group of Lie type, an inductive hypothesis yields the desired bound for $\left|\chi_{2}(x) / \chi_{2}(1)\right|$.

To estimate $\chi_{3}$, the results of [2] and Glauberman's character correspondence $[15,13.1]$ are used to show that if $\theta$ is an irreducible constituent of $\left(\chi_{3}\right)_{U_{J}}$, then $U_{J} / \operatorname{Ker} \theta$ is extraspecial. It is here that we must exclude the groups in characteristic 2 and 3 mentioned above. Standard Hall-Higman type results then yield that $\left|\chi_{3}(x) / \chi_{3}(1)\right| \leq$ $1 / q$.

Finally, $\chi_{4}$ is handled by restricting to $L_{J}$ and using an inductive hypothesis.

While our result is obviously not the last word on the problem of finding upper bounds for character values in groups of Lie type, we hope the reader will agree that our method offers a conceptual and effective approach to this problem.

I would like to thank P. Fong, P. Johnson, G. Seitz, and D. White for helpful conversations and correspondence related to this paper.

1. Preliminaries. This section contains preliminaries to our estimation of $\chi_{1}, \chi_{2}, \chi_{3}$, and $\chi_{4}$. Since we will apply an inductive hypothesis to Levi complements of parabolic subgroups, we must work with groups which are not necessarily quasisimple. Appropriate definitions and inductive machinery are introduced in this section. We also define the excluded "special" groups. The reason for their exclusion will not be apparent before $\S 3$. Finally, we estimate $|\chi(x) / \chi(1)|$ when $x$ is contained in no proper parabolic of $G$.

We begin with some important conventions. All fields $\operatorname{GF}(q)$ considered in this paper will have at least 4 elements. All algebraic groups $\bar{G}$ will be over the algebraic closure $\overline{\mathrm{GF}(p)}$ of the prime field $\mathrm{GF}(p)$. Algebraic groups and objects associated with them will be labeled with bars. We will denote by $\sigma$ an endomorphism of $\bar{G}$ such that $\bar{G}_{\sigma}$ is finite. Since $q \geq 4$, we then have $O^{p^{\prime}}\left(\bar{G}_{\sigma}\right)=\bar{G}_{\sigma}^{\prime}$. Following the usual convention, a simple algebraic group is semisimple with a simple root system and a possibly nontrivial center. A component of a finite group is a subnormal quasisimple subgroup.

DeFinition 1.1. Let $G$ be a finite group and $p$ a fixed prime num= ber. Let $x$ be a $p^{\prime}$-element of $G$. We say that $x$ is admissible if $x$ has prime order in $G / Z(G)$.

Definition 1.2. Let $\bar{G}$ be a simple algebraic group over $\overline{\mathrm{GF}(p)}$. Let $\sigma$ be an endomorphism of $\bar{G}$ such that $\bar{G}_{\sigma}$ is finite. We call $\bar{G}$ 
and $\bar{G}_{\sigma}$ special (following [2]) if $p=2$ and the root system of $\bar{G}$ is $B_{n}, C_{n}, F_{4}$ or $G_{2}$, or if $p=3$ and the root system of $\bar{G}$ is $G_{2}$.

Definition 1.3. Let $G$ be a finite group. We say $G$ is admissible if $G=\left\langle y, \bar{G}_{\sigma}^{\prime}\right\rangle$, where $\bar{G}$ is a connected reductive algebraic group of characteristic $p$ whose commutator subgroup is a product of simply connected and non-special simple components, and $y$ is an admissible element of $\bar{G}_{\sigma}$.

REMARK. In the definition above, we don't exclude the possibility that $y \in \bar{G}_{\sigma}^{\prime}$. Thus $G$ is either a central product of quasisimple groups of Lie type, or an extension of such a group by a cyclic group. The element $y$ then induces inner times diagonal automorphisms of the quasisimple factors of $\bar{G}_{\sigma}^{\prime}$. We note that an admissible group has a split $B N$-pair obtained by intersecting the $B$ and $N$ subgroups of $\bar{G}_{\sigma}$ with $G$. The parabolic subgroups of $G$ are also obtained from those of $\bar{G}_{\sigma}$ by intersection with $G$; see [3, p. 103].

Definition 1.4. Let $G$ be a finite group. We say that $G$ is simple admissible if $G$ is admissible and $G^{\prime}$ is quasisimple.

Definition 1.5. We say that $(G, x)$ is an admissible pair if $G$ is an admissible group and $x$ is an admissible element of $G$. If $G$ is also simple admissible, we say that $(G, x)$ is a simple admissible pair.

LEMMA 1.6. Let $G$ be an admissible group and let $L$ be a Levi complement of a parabolic subgroup of $G$. Let $K$ be a product of components of $L$. Let $y$ be an admissible element of $L$ such that $[y, K] \neq 1$. Then $\langle y, K\rangle$ is an admissible group.

Proof. Let $\bar{G}$ and $\sigma$ be as in Definition 1.3. Then $L=\bar{L}_{\sigma} \cap G$, where $\bar{L}$ is a $\sigma$-stable Levi complement in $\bar{G}$. Also $K=\bar{K}_{\sigma} \leq G$, where $\bar{K}$ is a product of simple components of $\bar{L}$. Let $\bar{T}$ be a $\sigma$ stable torus of $\bar{L}$ containing $y$. Then $\bar{K} \bar{T}$ is a connected reductive group with $\left((\bar{K} \bar{T})_{\sigma}\right)^{\prime}=K$. To complete the proof, it suffices to show that the simple components of $\bar{K}$ are simply connected. To prove this, we may assume that $\bar{G}^{\prime}$ is simple.

Let $\bar{L}_{1}$ be a simple component of $\bar{L}$. We may assume that $\bar{L}$ is a standard Levi subgroup $\bar{L}_{J}$, where $J$ is (by abuse) a subset of a fundamental set $\Pi$ for the root system $\bar{G}$. Then $\bar{L}_{1}$ corresponds to a connected subset $J_{1}$ of $J$.

Since $\bar{G}^{\prime}$ is simply connected, its diagonal subgroup $\bar{H}$ is the direct product of subgroups $\bar{H}_{\alpha}$, for $\alpha \in \Pi$, each isomorphic to the multiplicative group of $\overline{\mathrm{GF}(p)}$; see [7, pp. 197-198]. Let $\bar{H}_{1}$ be the direct 
product of the $\bar{H}_{\alpha}$ for $\alpha \in J_{1}$. Then $\bar{H}_{1}$ is the diagonal subgroup of $\bar{L}_{1}$. Let $\bar{M}_{1}$ be the simply connected covering group of $\bar{L}_{1}$. Let $\theta: \bar{M}_{1} \rightarrow \bar{L}_{1}$ be the natural epimorphism, as in [7, p. 190]. Clearly $\theta$ maps the diagonal subgroup of $\bar{M}_{1}$ isomorphically onto $\bar{H}_{1}$. It follows that $\operatorname{Ker} \theta=1$ and so $\bar{L}_{1}$ is simply connected.

LEMMA 1.7. Let $(G, x)$ be a simple admissible pair with rank $(G)>$ 1 . Suppose $x$ lies in a proper parabolic of $G$. Then for some standard maximal parabolic $P_{J}$ of $G, a$ G-conjugate of $x$ lies in $L_{J}$ and centralizes no component of $L_{J}$.

Proof. View $G$ as a group with a split $B N$-pair. Up to conjugacy, $x \in L_{J}$, the standard Levi complement of a standard maximal parabolic of $G$.

Suppose that $x$ centralizes every component of $L_{J}$. Then $x \in H$, the diagonal subgroup of $G$, and so $x \in L_{J^{\prime}}$, where $J^{\prime} \neq J$ is another maximal subset of the index set $I$ of the fundamental roots. If also $\left[x, L_{J^{\prime}}^{\prime}\right]=1$, then $x$ centralizes the standard Borel subgroup of $G$ and its "opposite", so $[x, G]=1$, a contradiction. Hence $x \in L_{J^{\prime}}$ and $x$ doesn't centralize every component of $L_{J^{\prime}}$.

Thus we may choose $J$ so that $x \in L_{J}$ and $x$ doesn't centralize every component of $L_{J}$. If $x$ centralizes no component of $L_{J}$, then we are done. Otherwise write $J=J_{1} \cup J_{2}$, where $J_{1}$ corresponds to the union of the components of $L_{J}$ centralized by $x$ and $J_{2}$ corresponds to the union of the components of $L_{J}$ not centralized by $x$. Write $x=h x_{1} x_{2}$, with $h \in H, x_{1} \in L_{J_{1}}^{\prime}$ and $x_{2} \in L_{J_{2}}^{\prime}$. Then $h x_{1}$ centralizes $L_{J_{1}}^{\prime}$. Since $h$ normalizes every root subgroup of $L_{J_{1}}$, so does $x_{1}=h^{-1}\left(h x_{1}\right)$. It follows that $x_{1} \in H$.

Thus $x=k x_{2}$, with $k \in H$ and $x_{2} \in L_{J_{2}}^{\prime}$. Thus $x \in L_{J_{2}}$. If $J_{2}$ is connected, we may choose a maximal and connected subset $J_{3}$ of $I$ with $J_{2} \leq J_{3}$. If $J_{2}$ is not connected, then $J$ has 3 components, the root system of $G$ is $D_{n}, E_{6}, E_{7}$, or $E_{8}$, and one checks that it is still possible to choose a maximal and connected subset $J_{3}$ of $I$ with $J_{2} \leq J_{3}$.

Hence $x \in L_{J_{3}}$, and since $x$ doesn't centralize $L_{J_{2}}^{\prime} \leq L_{J_{3}}^{\prime}, x$ doesn't centralize the unique component of $L_{J_{3}}$.

Definition 1.8. Let $(G, x)$ be a simple admissible pair. Let $L_{J}$ be the standard Levi complement of a standard maximal parabolic of $G$. Suppose $x \in L_{J}$ and $x$ centralizes no component of $L_{J}$. Then $\left(G, x, L_{J}\right)$ is called an admissible triple. 
We now turn to the problem of estimating $|\chi(x) / \chi(1)|$ when $x$ lies in no proper parabolic of $G$.

Lemma 1.9. Let $(G, x)$ be a simple admissible pair. Suppose $x$ is contained in no proper parabolic of $G$. Then $C_{G^{\prime}}(x)$ is a maximal torus of $G^{\prime}$.

Proof. By the Borel-Tits theorem [3, p. 103], $C_{G}(x)$ contains no unipotent elements. Let $\bar{G}$ and $\sigma$ be as in Definition 1.3. Since $C_{\bar{G}}(x)$ admits $\sigma$, it follows that $C_{\bar{G}}(x)$ contains no unipotent elements. By a theorem of Steinberg $[8,3.5 .6], C_{\bar{G}}(x)$ is connected, and so is a (necessarily maximal) torus $\bar{T}$ of $\bar{G}$. By $\left[8\right.$, p. 88], $\bar{T} \cap \bar{G}^{\prime}$ is a maximal torus of $\bar{G}^{\prime}$, and so taking $\sigma$-fixed points yields that $C_{G^{\prime}}(x)$ is a maximal torus of $G^{\prime}$.

LEMMA 1.10. Let $(G, x)$ be an admissible pair with $G^{\prime}=\operatorname{SL}(2, q)$. Let $\chi \in \operatorname{Irr}(G)$ be nonlinear. Then $|\chi(x) / \chi(1)|=\left|\psi\left(x_{1}\right) / \psi(1)\right|$, where $\psi$ is an irreducible character of $\operatorname{SL}(2, q)$ or $\mathrm{GL}(2, q)$ and $x_{1}$ is a noncentral semisimple element of $\mathrm{SL}(2, q)$ (resp. $\mathrm{GL}(2, q))$, and $\psi(1)=\chi(1)$.

Proof. Let $G=\left\langle G^{\prime}, y\right\rangle$, as in Definition 1.3. Suppose $y$ induces an inner automorphism of $G^{\prime}$. Let $g \in G^{\prime}$ induce the same automorphism of $G^{\prime}$ as $y$. Let $z=y g^{-1}$ and let $r$ be the prime order of $y \bmod Z(G)$. Then $z^{r} \equiv y^{r}\left(\bmod G^{\prime}\right)$, and so $z^{r} \in G^{\prime} O_{p^{\prime}}(Z(G))$. Since $z \in Z(G), z^{r} \in\left(G^{\prime} O_{p^{\prime}}(Z(G))\right) \cap Z(G) \leq O_{p^{\prime}}(Z(G))$. Thus $G$ is a central product $G^{\prime}\langle z\rangle$, where $z$ is a $p^{\prime}$-element. The conclusion of the lemma follows with $\psi \in \operatorname{Irr}(\operatorname{SL}(2, q))$.

Next suppose $y$ induces an outer automorphism $\alpha$ of $G^{\prime}$. Then $q$ is odd. Since $y$ has prime order $\bmod Z(G)$ and the diagonal automorphism group of $G^{\prime}$ has order 2, we have $\alpha^{2}=1$. Let $H$ be the semidirect product $\langle\alpha\rangle G^{\prime}=\langle\alpha\rangle \operatorname{SL}(2, q)$. If we can find abelian $p^{\prime}$ groups $Z, Z_{1}, W$, and $W_{1}$ such that $G * Z \cong H * W$ and $\operatorname{GL}(2, q) *$ $Z_{1} \cong H * W_{1}$, where $*$ denotes a central product, then the conclusion of the lemma follows with $\psi \in \operatorname{Irr}(\operatorname{GL}(2, q))$.

To do this, let $Z=\langle z\rangle$ be a cyclic group of order $|y|$. Form the central product $G * Z$, where $z^{2}=y^{2} \in Z(G)$. Then $y z^{-1}$ is an involution which induces $\alpha$ on $G^{\prime}$, and $G * Z=\left(\left\langle y z^{-1}\right\rangle G^{\prime}\right) * Z \cong H * Z$. Next let $t \in \operatorname{GL}(2, q)$ induce $\alpha$ on $\operatorname{SL}(2, q)$. Then $t$ is a semisimple element. Let $Z_{1}=\left\langle z_{1}\right\rangle$ be a cyclic group of order $|t|$ and form the central product $\operatorname{GL}(2, q) * Z_{1}$, where $z_{1}^{2}=t^{2} \in Z(\mathrm{GL}(2, q))$. 
Then $z_{1} t^{-1}$ is an involution which induces $\alpha$ on $\operatorname{SL}(2, q)$, and so $\mathrm{GL}(2, q) * Z_{1} \cong\left(\left\langle z_{1} t^{-1}\right\rangle \mathrm{SL}(2, q)\right) *\left(Z(\mathrm{GL}(2, q)) Z_{1}\right)=H * W_{1}$.

Theorem 1.11. Let $(G, x)$ be a simple admissible pair. Suppose that $x$ is contained in no proper parabolic of $G$. Let $\chi \in \operatorname{Irr}(G)$ with $\chi(1)>1$. Then $|\chi(x) / \chi(1)| \leq 6 / q$.

Proof. If $G^{\prime}=\operatorname{SL}(2, q)$, then Lemma 1.10 implies that $|\chi(x) / \chi(1)|$ $=\left|\psi\left(x_{1}\right) / \psi(1)\right|$, as in the conclusion of Lemma 1.10. Checking character tables $([\mathbf{1 1}],[\mathbf{1 8}])$ shows that the last ratio is at most $2 /(q-1) \leq 6 / q$. Hence we assume $G^{\prime} \neq \mathrm{SL}(2, q)$.

We claim that there exists a group $H$ and an element $h \in H$ such that $H^{\prime}=G^{\prime}, C_{G^{\prime}}(h)=C_{G^{\prime}}(x),\{|\chi(x) / \chi(1)|: \chi \in \operatorname{Irr}(G)$ and $\chi(1)>$ $1\}=\{|\psi(h) / \psi(1)|: \psi \in \operatorname{Irr}(H)$ and $\psi(1)>1\}$, and $\left|H: G^{\prime}\right| \leq d$, where $d$ is the order of the diagonal automorphism group of $G^{\prime}$.

Write $G=\left\langle y, \bar{G}_{\sigma}^{\prime}\right\rangle$, as in Definition 1.3. Clearly $\bar{G}_{\sigma}^{\prime}=G^{\prime}$, so $G=\langle y\rangle G^{\prime}$. To prove the claim we may assume that $y \notin G^{\prime}$.

Let $Z=\langle z\rangle$ be a cyclic group of order $|y|$. Let $r$ be the prime order of $y \bmod Z(G)$. Form the central product $G * Z$, where $z^{r}=$ $y^{r} \in Z(G)$. Then $\left|y z^{-1}\right|=r$ and $G * Z=\left(\left\langle y z^{-1}\right\rangle G^{\prime}\right) * Z$. Let $G^{*}=\left\langle y z^{-1}\right\rangle G^{\prime}$. Write $x=x_{1} w$ where $x_{1} \in G^{*}$ and $w \in Z$. Then $\left(G^{*}\right)^{\prime}=G^{\prime}, C_{G^{\prime}}(x)=C_{G^{\prime}}\left(x_{1}\right)$, and $\{\chi(x) / \chi(1) \mid: \chi \in \operatorname{Irr}(G)$ and $\chi(1)>1\}=\left\{\zeta\left(x_{1}\right) / \zeta(1) \mid: \zeta \in \operatorname{Irr}\left(G^{*}\right)\right.$ and $\left.\zeta(1)>1\right\}$.

If $y z^{-1}$ induces an outer automorphism of $G^{\prime}$, then since $y z^{-1}$ has prime order $r$, we have $r \mid d$. Hence our claim holds for $H=G^{*}$, $h=x_{1}$.

We therefore assume that $y z^{-1}$ induces an inner automorphism of $G^{\prime}$. If $r$ divides $\left|Z\left(G^{\prime}\right)\right|=d$, the claim holds as above. Hence we assume $\left(r,\left|Z\left(G^{\prime}\right)\right|\right)=1$. For some element $v$ in the coset $y z^{-1} G^{\prime}, G^{*}$ is a central product $G^{\prime}\langle v\rangle$. We have $v^{r} \in G^{\prime} \cap Z(G) \leq Z\left(G^{\prime}\right)$. Since $r$ doesn't divide $\left|Z\left(G^{\prime}\right)\right|$, we have $\left|O_{r}(\langle v\rangle)\right|=r$ and $G^{*}=$ $G^{\prime}\left(O_{r}(\langle v\rangle) \times O_{r^{\prime}}(\langle v\rangle)\right)=G^{\prime} \times O_{r}(\langle v\rangle)$. Write $x_{1}=g u$, with $g \in G^{\prime}$ and $u \in O_{r}(\langle v\rangle)$. Then $C_{G^{\prime}}\left(x_{1}\right)=C_{G^{\prime}}(g)$ and $\left\{\left|\zeta\left(x_{1}\right) / \zeta(1)\right|: \zeta \in \operatorname{Irr}\left(G^{*}\right)\right.$ and $\zeta(1)>1\}=\left\{|\psi(g) / \psi(1)|: \psi \in \operatorname{Irr}\left(G^{\prime}\right)\right.$ and $\left.\psi(1)>1\right\}$. Hence our claim holds with $H=G^{\prime}, h=g$. This proves the claim in all cases.

To prove the theorem, it suffices to show that $|\psi(h) / \psi(1)| \leq 6 / q$ for any nonlinear irreducible character $\psi$ of $H$. Using Lemma 1.9, $|\psi(h)|^{2} \leq\left|C_{H}(h)\right| \leq\left|C_{G^{\prime}}(h)\right| d=\left|C_{G^{\prime}}(x)\right| d=|T| d$, where $T$ is a torus of $G^{\prime}$. By the order formula for tori (see [8, p. 98]), $|T| \leq(q+1)^{l}$, 
where $l$ is the rank of $\bar{G}$, which is greater than the rank of $G$ when $G$ is twisted. Since $G^{\prime} \neq \operatorname{SL}(2, q), l>1$.

We have $d \leq l+1$. By [16, p. 419], $\psi(1) \geq\left(q^{l}-1\right) / 2$. Then

$$
\frac{|\psi(h)|}{\psi(1)} \leq \frac{2 \sqrt{l+1}(q+1)^{l / 2}}{q^{l}-1} \leq \frac{32 \sqrt{l+1}(q+1)^{l / 2}}{15\left(q^{2}-1\right)^{l / 2}}=\frac{32 \sqrt{l+1}}{15(q-1)^{l / 2}} \leq \frac{6}{q}
$$

for $l \geq 2$ and $q \geq 4$. The second inequality uses $\left(q^{l}-1\right) /\left(q^{2}-1\right)^{l / 2} \geq$ $\left(q^{l}-1\right) /\left(q^{2}\right)^{l / 2}=1-q^{-l} \geq 15 / 16$. The third inequality holds because $\sqrt{l+1} /(q-1)^{l / 2}$ is decreasing in $q$ and $l$ for $l \geq 2$ and $q \geq 4$.

2. Estimating $\chi_{1}$. Let $G$ be a simple admissible group and let $P_{J}$ be a standard maximal parabolic of $G$. Let $\chi \in \operatorname{Irr}(G)$ and let $\lambda$ be a linear character of $P_{J}$. In the first part of this section we establish an absolute upper bound for the multiplicity $\left(\chi_{P_{J}}, \lambda\right)$.

Our work will be based on the following "comparison theorem" of McGovern [17]. By abuse, we will use the same symbol $\lambda$ to denote the restriction of $\lambda$ to the diagonal subgroup of $G$. Since $q \geq 4$, this diagonal subgroup covers $P_{J} / P_{J}^{\prime}$ and $L_{J} / L_{J}^{\prime}$. Let $W(\lambda)$ be the stabilizer of $\lambda$ in $W$, the Weyl group of $G$. In [17], a certain set $D(\lambda, J)$ of $\left(W(\lambda), W_{J}\right)$-double coset representatives is defined. We may assume that $1 \in D(\lambda, J)$.

THEOREM 2.1. Let $G$ be a finite group with a split $B N$-pair of charcteristic $p$. There is a one-to-one correspondence between the constituents of $\lambda_{B}^{G}$ and the irreducible characters of $W(\lambda)$. Suppose $\chi$ is the constituent of $\lambda_{B}^{G}$ corresponding to $\phi \in \operatorname{Irr}(W(\lambda))$. Let $\kappa$ be an irreducible character of $L_{J}$ with inflation $\tilde{\kappa}$ to $P_{J}$, satisfying $\left(\chi, \tilde{\kappa}^{G}\right) \neq 0$. Then $\kappa$ is a constituent of $\left(\lambda^{v}\right)_{B_{J}}^{L_{J}}$ and $\tilde{\kappa}$ is a constituent of $\left(\lambda^{v}\right)_{B}^{P_{J}}$ for a unique $v \in D(\lambda, J)$. Both $\kappa$ and $\tilde{\kappa}$ correspond to the same unique irreducible character $\psi$ of $W_{J} \cap W\left(\lambda^{v}\right)$, and $\left(\chi, \tilde{\kappa}^{G}\right)=\left(\phi^{v}, \psi^{W\left(\lambda^{v}\right)}\right)$.

Proof. This is [17, Theorem A] with some minor changes in wording. In the statement of this theorem, $\lambda$ denotes an arbitrary linear character of the diagonal subgroup of $G$. Note that $1 \in W$ is " $\lambda$ special" ([17, p. 426]), so we may assume $1 \in D(\lambda, J)$.

We will apply Theorem 2.1 with $\kappa=\lambda_{L_{J}}, \tilde{\kappa}=\lambda$. Then the multiplicity we wish to bound, $\left(\chi, \lambda^{G}\right)$, equals $\left(\phi^{v}, \psi^{W\left(\lambda^{v}\right)}\right)$, where $\psi \in \operatorname{Irr}\left(W_{J} \cap W\left(\lambda^{v}\right)\right)$ corresponds to $\lambda$ and $\lambda_{L_{J}}$. We will show that $v=1$ and $\psi=1$. Since $W_{J} \cap W(\lambda)=W_{J}$ by Lemma 2.2 below, we 
will need only to establish an absolute upper bound for $\left(\phi, 1_{W_{J}}^{W(\lambda)}\right)$ as $\phi$ ranges over $\operatorname{Irr}(W(\lambda))$. But this reduces to bounding the multiplicities in $1_{W_{J}}^{W}$, which have been investigated in [9] and [1].

LEMMA 2.2. With notation as above, we have $W_{J} \leq W(\lambda)$ and $v=1$.

Proof. Since $N_{J} \leq P_{J}$ stabilizes $\lambda \in \operatorname{Irr}\left(P_{J}\right)$, we have $W_{J} \leq W(\lambda)$. Since $\kappa=\lambda_{L_{J}}$ is a constituent of $\lambda_{B_{J}}^{L_{J}}$ and $\tilde{\kappa}=\lambda$ is a constituent of $\lambda_{B}^{P_{J}}$, uniqueness in Theorem 2.1 implies that $v=1$.

The next remark, which is copied from [17, p. 421], summarizes some of the main results of [13]. Here $\Phi$ is the root system of $G$, with fundamental system $\Pi$.

REMARK 2.3. Let $G$ be a group with a split $B N$-pair of characteristic $p$. Let $\lambda$ be a linear character of the diagonal subgroup of $G$. Define the $\lambda$-parameters $q_{a}(\lambda)$ as in [13]. There is a prime power $q$ of $p$ such that $q_{a}(\lambda)=q^{c_{a}(\lambda)}$ for all roots $a$ in the root system $\Phi$ of $W$. In case $G=G(q)$ is a finite group of Lie type, this prime power may be taken as the characteristic power $q$ of $G$. Howlett and Kilmoyer proved that there is a semidirect product decomposition $W(\lambda)=A C$, where $C$ is the reflection group with root system $\Gamma=\left\{a \in \Phi: q_{a}(\lambda) \neq 1\right\}$ and fundamental system $\Sigma \leq \Phi^{+}$, and $A$ is an abelian $p^{\prime}$-group which normalizes $C$. Then a generic algebra $A(\lambda)$ was constructed, which is an associative $\bar{Q}[t]$-algebra with basis $\left\{X_{w}: w \in W(\lambda)\right\}$, satisfying the following multiplication (for $a \in A, b \in \Sigma$, and $w \in W(\lambda))$ :

$$
\begin{aligned}
X_{a} X_{w} & =X_{a w}, \quad X_{w} X_{a}=X_{w a}, \\
X_{w} X_{w_{b}} & = \begin{cases}X_{w w_{b}} & \text { if } w(b) \in \Gamma^{+}, \\
t_{b}(\lambda) X_{w w_{b}}+\left(t_{b}(\lambda)-1\right) X_{w} & \text { if } w(b) \in \Gamma^{-},\end{cases} \\
X_{w_{b}} X_{w} & = \begin{cases}X_{w_{b} w} & \text { if } w^{-1}(b) \in \Gamma^{+}, \\
t_{b}(\lambda) X_{w_{b} w}+\left(t_{b}(\lambda)-1\right) X_{w} & \text { if } w^{-1}(b) \in \Gamma^{-},\end{cases}
\end{aligned}
$$

where $t_{b}(\lambda)=t^{c_{b}(\lambda)}$.

Definition. In the situation of Remark 2.3, let $W_{J}(\lambda)=W_{J} \cap$ $W(\lambda)$. Let $A_{J}(\lambda)$ be the generic algebra associated with $W_{J}(\lambda)$. (Note that $W_{J}(\lambda)$ is the stabilizer of $\lambda$ in the Weyl group of $L_{J}$, so it has an associated generic algebra as in Remark 2.3.)

LeMma 2.4. Let $G, P_{J}$ and $\lambda$ be as in Lemma 2.2. Let $q_{a}(\lambda)$ be as in Remark 2.3. Then $q_{a}(\lambda)=q_{a}(1)$ for $a \in \Phi_{J}$. 
Proof. For any $a \in \Phi,[13,2.6(\mathrm{~b})]$ says that $q_{a}(\lambda)+q_{a}(\lambda)^{-1}=$ $\left[\sum \lambda^{w}\left(h_{i}(x)\right)\right]^{2} \lambda^{w}\left(\left(r_{i}\right)^{2}\right)$, whenever $w \in W$ is chosen so that $a^{w} \in \Pi$. By $\left[13\right.$, p. 577], $h_{i}(x)$ and $\left(r_{i}\right)^{2}$, which are independent of $\lambda$, may be chosen to lie in the diagonal subgroup of $O^{p^{\prime}}\left(L_{i}\right)$, where $L_{i}$ is the standard Levi complement of the standard minimal parabolic of $G$ corresponding to $\left\{a^{w}\right\} \leq \Pi$.

Since $a \in \Phi_{J}$ by hypothesis, we may take $w \in W_{J}$, so that $a^{w} \in$ $\Pi_{J}$. Then $L_{i} \leq L_{J}$. Since $O^{p^{\prime}}\left(L_{i}\right) \leq O^{p^{\prime}}\left(P_{J}\right)=P_{J}^{\prime} \leq \operatorname{Ker} \lambda$, we have $q_{a}(\lambda)+q_{q}(\lambda)^{-1}=q_{a}(1)+q_{a}(1)^{-1}$. By $[13, \S 4]$, for any $a \in \Phi, q_{a}(\lambda)=$ $q^{c_{a}(\lambda)}$, where $c_{a}(\lambda)$ is a non-negative integer. Thus $q_{a}(\lambda)=q_{a}(1)$.

REMARK 2.5. Let $a \in \Phi$ and choose $w \in W$ so that $a^{w} \in \Pi$. Let $s$ be the fundamental reflection corresponding to $a^{w}$. Then $q_{a}(1)=$ ind $s=\left|B: B \cap B^{s}\right|$, the usual index parameter defined in [10, p. 610]. See [13, p. 552]. If $a \in \Phi_{J}$, then $q_{a}(\lambda)$ is the same, whether computed in $G$ or $L_{J}$.

LeMma 2.6. Let $G, P_{J}$, and $\lambda$ be as in Lemma 2.2. Write $W_{J}(\lambda)=$ $A_{J} C_{J}$, as in Remark 2.3. Then $C_{J}=W_{J}=W_{J}(\lambda)$ and $A_{J}=1$. The generic algebra $A_{J}(\lambda)$ can be identified with the generic algebra of the Coxeter system $\left(W_{J}, \Pi_{J}\right)$ over $\bar{Q}[t]$.

Proof. By [17, Theorem 1.5], the reflection factor $C_{J}$ of $W_{J}(\lambda)$ equals $W_{J} \cap C$, where $C$ is the reflection factor of $W(\lambda)$. Since the root system of $C$ contains $\Phi_{J}$ by Lemma 2.4 , we have $W_{J} \leq C$, proving the first assertion. The second assertion is clear from the definitions of the respective generic algebras in Remark 2.3 and [10, p. 637], and the fact that $c_{a}(\lambda)=c_{a}(1)$ for $a \in \Phi_{J}$ by Lemma 2.4.

Proposition 2.7. Let $G, P_{J}$, and $\lambda$ be as in Lemma 2.2. Let $\psi \in \operatorname{Irr}\left(W_{J}\right)$ correspond to $\kappa=\lambda_{L_{J}}$ in Theorem 2.1. Then $\psi=1_{W_{J}}$.

Proof. By [17, p. 431], the bijection between the irreducible characters of the Hecke algebra $\mathscr{H}\left(L_{J}, B_{J}, \lambda\right)$ and those of $\bar{Q}\left[W_{J}\right]$ is obtained via [17, Theorem 2.1]. An irreducible character of one of these algebras corresponds to an irreducible character of the other when both are (extended) specializations of the same irreducible character of $A_{J}(\lambda)$.

Following [10, p. 637], we define a $\bar{Q}[t]$-algebra homomorphism $\mathrm{IND}: A_{J}(\lambda) \rightarrow \bar{Q}[t]$ by $\operatorname{IND} X_{s_{l}}=t^{c_{a_{l}}(\lambda)}=t_{a_{t}}(\lambda)$, where $a_{i}$ is a 
fundamental root in $\Pi_{J}$ and $s_{i}$ is the corresponding fundamental reflection. Clearly IND specializes to the principal character of character of $\bar{Q}\left[W_{J}\right]$ under the specialization $t \rightarrow 1$. To compute the specialization of IND to $\mathscr{H}\left(L_{J}, B_{J}, \lambda\right)$, we must check that the specialization $t \rightarrow q$ sends the generator $X_{s_{i}}$ of $A_{J}(\lambda)$ to the element $\beta_{s_{t}}=$ (ind $\left.s_{i}\right) e_{\lambda}\left(s_{i}\right) e_{\lambda}$, where $s_{i} \in W_{J}$ is a fundamental reflection, $\left(s_{i}\right) \in$ $N_{J} \cap L_{J}^{\prime}$ maps onto $s_{i}$, and $e_{\lambda}$ is the primitive central idempotent of $\bar{Q}\left[B_{J}\right]$ corresponding to $\lambda$. By Theorem 2.17 and p. 567 of [13], $X_{s_{i}}$ is sent to $\gamma_{s_{t}} \in \mathscr{H}\left(L_{J}, B_{J}, \lambda\right)$, where $\gamma_{s_{i}}=\lambda\left(\left(s_{i}\right)\right) q_{s_{i}}^{-1 / 2} q_{s_{i}}(\lambda)^{1 / 2} \beta_{s_{i}}$ by [13, Eq. 2.19]. Also $q_{s_{i}}=q_{a_{i}}(1)$ and $q_{s_{i}}(\lambda)=q_{a_{i}}(\lambda)$ by the definitions in [13]. By Lemma 2.14 and the fact that $\left(s_{i}\right) \in L_{J}^{\prime}$, we have $\gamma_{s_{i}}=\beta_{s_{i}}$. (Note that the definition of $\beta_{w}$ in [13, Def. 2.2] is incorrect. The given formula for $\beta_{w}$ must be multiplied by ind $w$, since otherwise [13, Theorem 2.4] would be incorrect and equation 2.19 would be inconsistent with Theorem 2.17.)

Let ind: $\mathscr{H}\left(L_{J}, B_{J}, \lambda\right) \rightarrow \bar{Q}$ be the specialization of IND under $t \rightarrow q$. Then $\operatorname{ind}\left(\beta_{s_{i}}\right)$ is the specialization of $t^{c_{a_{i}}(\lambda)}$ under $t \rightarrow q$. Hence $\operatorname{ind}\left(\beta_{s_{i}}\right)=q_{a_{i}}(\lambda)=q_{a_{i}}(1)=$ ind $s_{i}$, as in Remark 2.5. On the other hand, $\lambda\left(\beta_{s_{l}}\right)=\left(\right.$ ind $\left.s_{i}\right) \lambda\left(e_{\lambda}\right) \lambda\left(\left(s_{i}\right)\right) \lambda\left(e_{\lambda}\right)=$ ind $s_{i}$. Since the $\beta_{s_{t}}$ generate $\mathscr{H}\left(L_{J}, B_{J}, \lambda\right)$ as a $\bar{Q}$-algebra, ind equals the restriction of $\lambda$ to $\mathscr{H}\left(L_{J}, B_{J}, \lambda\right)$, so $\lambda_{L_{J}}$ corresponds to the principal character of $W_{J}$, as desired.

Corollary 2.8. Let $G, P_{J}$, and $\lambda$ be as in Lemma 2.2. Let $M(\lambda)=\max \left\{\left(\lambda^{G}, \chi\right): \chi \in \operatorname{Irr}(G)\right\}$. Let $m(W, J)=\max \left\{\left(1_{W_{J}}^{W}, \phi\right)\right.$ : $\phi \in \operatorname{Irr}(W)\}$, where $W$ is the Weyl group of $G$. Then $M(\lambda) \leq$ $m(W, J)$.

Proof. Choose $\chi \in \operatorname{Irr}(G)$ so that $\left(\lambda^{G}, \chi\right)=M(\lambda)$. Let $\phi \in$ $\operatorname{Irr}(W(\lambda))$ correspond to $\chi$ in Theorem 2.1. By Theorem 2.1 and Proposition 2.7, $1_{W_{J}}^{W(\lambda)}=M(\lambda) \phi+\alpha$, where $\alpha$ is a sum of other irreducible characters of $W(\lambda)$. Thus some irreducible constituent of $1_{W_{J}}^{W}$ has multiplicity at least $M(\lambda)$, as desired.

It now suffices to show that there is an absolute upper bound for $m(W, J)$ as $W$ ranges over all irreducible Weyl groups and $W_{J}$ ranges over all maximal parabolic subgroups of $W$. For $W$ of type $A_{n}, B_{n}$, or $C_{n}, m(W, J)=1$ for all $J$ by $[9$, p. 90]. For the exceptional Weyl groups, Alvis' tables [1] yield $m(W, J) \leq 13$ for all $W$ and $J$, with 13 occurring for $W\left(E_{8}\right)$. 
It remains to determine $m(W, J)$ for $W$ of type $D_{n}$. By [9, p. 90], $m\left(W\left(D_{5}\right), J\right)>1$ for at least one $J$.

Proposition 2.9. $m\left(W\left(D_{n}\right), J\right) \leq 2$ for all $n$ and $J$.

Proof. Let $W=W\left(D_{n}\right)$. Let $Y=W\left(B_{n}\right)$. Then $W \leq Y \leq$ $S_{n} E_{n}$, where $S_{n}$ is the symmetric group of degree $n$, and $E_{n}$ is an elementary abelian group of order $2^{n}$ on which $S_{n}$ acts by permuting coordinates. Write elements of $E_{n}$ as row vectors with entries in $\mathrm{GF}(2)$. For $1 \leq i \leq n-1$, let $w_{i}=(i, i+1) \in S_{n}$. Let $w_{n}=$ $w_{n-1}[00 \cdots 011]$ and let $y_{n}=[00 \cdots 01]$. Let $y_{i}=w_{i}$ for $1 \leq i \leq$ $n-1$. Then $\left\{w_{1}, \ldots, w_{n}\right\}$ and $\left\{y_{1}, \ldots, y_{n}\right\}$ are standard sets of fundamental reflections for $W$ and $Y$, respectively.

Let $J=\{1, \ldots, n\}-\{j\}$. If $j \leq n-2$, then $\left|Y_{J}: W_{J}\right|=2$, $W Y_{J}=Y$, and $Y_{J} \cap W=W_{J}$. By Mackey's theorem, $\left(1_{Y_{J}}^{Y}\right)_{W}=$ $1_{W_{J}}^{W}$. By [9, p. 90], $1_{Y_{J}}^{Y}$ is a sum $\phi_{1}+\cdots+\phi_{k}$ of distinct irreducible characters of $Y$. If $1 \leq i<r \leq k$, then $\left(\phi_{i}\right)_{W}$ and $\left(\phi_{r}\right)_{W}$ have a common irreducible constituent if and only if $\phi_{i}=\mu \phi_{r}$, where $\mu$ is the nonprincipal linear character of $Y / W$. Since each $\left(\phi_{i}\right)_{W}$ is either irreducible or is the sum of two distinct $Y$-conjugate irreducible characters, it follows that all multiplicities in $1_{W_{J}}^{W}$ are at most 2.

If $j=n-1$, then $Y_{J} \cap W$ is the non-maximal standard parabolic subgroup of $W$ corresponding to $\{1, \ldots, n-2\}$ and $Y_{J} W=Y$. The argument in the preceding paragraph shows that the multiplicities in $1_{Y_{J} \cap W}^{W}$ are at most 2. Since $Y_{J} \cap W \leq W_{J}$, it follows that the multiplicities in $1_{W_{J}}^{W}$ are at most 2.

If $j=n$, then $W_{J}=Y_{J}$. Since $1_{Y_{J}}^{Y}$ is multiplicity-free, so is $1_{Y_{J}}^{W}=1_{W_{J}}^{W}$.

We summarize our work on multiplicities in the following theorem.

THeORem 2.10. Let $G$ be a simple admissible group. Let $P_{J}$ be a maximal parabolic subgroup of $G$. Let $\chi \in \operatorname{Irr}(G)$ and let $\lambda$ be a linear character of $P_{J}$. Then $\left(\chi_{P_{J}}, \lambda\right) \leq 13$.

Proof. This follows from Theorem 2.1, Proposition 2.7, Corollary 2.8, Proposition 2.9, and the remarks preceding Proposition 2.9.

Let $G, \chi$, and $P_{J}$ be as in Lemma 2.2. Having bounded the murtiplicities of the linear constituents of $\chi_{P_{J}}$, we must now bound the 
number of distinct linear constituents of $\chi_{P_{J}}$. Linear characters of $P_{J}$ are cuspidal characters of the diagonal subgroup of $G$. Hence if $\lambda$ and $\mu$ are two linear constituents of $\chi_{P_{J}}$, Harish-Chandra's theorem [10,70.15(A)] implies that $\lambda$ and $\mu$, viewed as characters of the diagonal group of $G$, are conjugate under $W$. Thus we must bound the number $n(\lambda)$ of linear characters of $P_{J}$ which are $W$-conjugate to a fixed linear character $\lambda$ of $P_{J}$.

Let $P_{0}=P_{J} \cap G^{\prime}$. Then $P_{0}$ is a maximal parabolic of $G^{\prime}$. Let $H$ denote the diagonal subgroup of $G^{\prime}$. Let $\lambda^{\prime}$ be the restriction of $\lambda$ to $P_{0}$. We may also view $\lambda^{\prime}$ as a linear character of $H$. The semidirect product decomposition in Remark 2.3 applies to both $W(\lambda)$ and $W\left(\lambda^{\prime}\right)$. Write $W(\lambda)=A(\lambda) C(\lambda)$ and $W\left(\lambda^{\prime}\right)=A\left(\lambda^{\prime}\right) C\left(\lambda^{\prime}\right)$. Let $\widehat{C}(\lambda)$ be the group generated by all reflections in $W(\lambda)$, so that $C(\lambda) \leq$ $\widehat{C}(\lambda) \leq W(\lambda)$. Define $\widehat{C}\left(\lambda^{\prime}\right)$ similarly.

We will use the fact, proved below, that $P_{0}$ has a cyclic commutator factor group to reduce the problem of bounding $n(\lambda)$ to known results on the indices of reflection subgroups of $W$ in their normalizers.

Lemma 2.11. Let $P_{0}$ and $H$ be as above. Then $P_{0} / P_{0}^{\prime}$ is cyclic.

Proof. Since $[H, X]=X$ for every root subgroup $X$ of $G^{\prime}$, it follows that $P_{0}^{\prime}=O^{p^{\prime}}\left(P_{0}\right)$ and $P_{0}=P_{0}^{\prime} H$. Hence $P_{0} / P_{0}^{\prime} \cong$ $H /\left(H \cap P_{0}^{\prime}\right)$.

First suppose $G^{\prime}$ is untwisted. Then, since $G^{\prime}$ is simply connected by hypothesis, $H$ is the direct product of groups $H_{\alpha}$, each isomorphic to the multiplicative group of $\operatorname{GF}(q)$. See [7, p. 197-8]. Moreover, the $H_{\alpha}$ correspond to the fundamental roots $\alpha \in \Pi$. By [7, p. 92], $H_{\alpha} \leq\left\langle X_{\alpha}, X_{-\alpha}\right\rangle$. Hence $\prod_{\alpha \in J} H_{\alpha} \leq O^{p^{\prime}}\left(P_{0}\right)=P_{0}^{\prime}$. Thus $H /\left(H \cap P_{0}^{\prime}\right)$ is cyclic.

Next suppose $G^{\prime}$ is a twisted group over $\operatorname{GF}(q)$. Write $G^{\prime}=\bar{G}_{\sigma}$, where $\bar{G}$ is a simply connected algebraic group over $\overline{\mathrm{GF}(p)}$, and $\sigma=$ $q \tau$, where $q$ is the $q$ th power Frobenius endomorphism of $\bar{G}$, and $\tau$ is a nontrivial graph automorphism. Let $P_{0}=\bar{P}_{\sigma}$, where $\bar{P}$ is a standard $\sigma$-stable parabolic of $\bar{G}$. Since $\bar{G}$ is a simply connected Chevalley group over $\overline{\mathrm{GF}(p)}$, its diagonal subgroup $\bar{H}$ is a direct product of subgroups $\bar{H}_{\alpha}$, where $\alpha$ ranges over a fundamental set of roots for $\bar{G}$ and each $\bar{H}_{\alpha}$ is isomorphic to the multiplicative group of $\overline{\mathrm{GF}(p)}$. As in the preceding paragraph, we may write $\bar{H}=\bar{H}_{1} \times \bar{H}_{2}$, where $\bar{H}_{2} \leq \bar{P}^{\prime}$ and $\bar{H}_{1}$ is the direct product of the $\bar{H}_{\alpha}$ as $\alpha$ ranges over a single $\tau$-orbit of fundamental roots of $\bar{G}$. Then $H=\bar{H}_{\sigma}=$ 
$\left(\bar{H}_{1}\right)_{\sigma} \times\left(\bar{H}_{2}\right)_{\sigma}$ and $\left(\bar{H}_{2}\right)_{\sigma} \leq H \cap\left(\bar{P}^{\prime}\right)_{\sigma}=H \cap P_{0}^{\prime}$. Hence it suffices to show that $\left(\bar{H}_{1}\right)_{\sigma}$ is cyclic. Let $\pi: \bar{H}_{1} \rightarrow(\overline{\mathrm{GF}(p)})^{*}$ be the projection onto one fixed direct factor of $\bar{H}_{1}$. Since $\tau$ cyclically permutes the direct factors of $\bar{H}_{1}$ and $\sigma=q \tau$, it follows that the restriction of $\pi$ to $\left(\bar{H}_{1}\right)_{\sigma}$ is injective, and so $\left(\bar{H}_{1}\right)_{\sigma}$ is cyclic.

LEMMA 2.12. Let $n(\lambda)$ and $\lambda^{\prime}$ be as in the remarks preceding Lemma 2.11. Then $n(\lambda) \leq\left|N_{W}\left(\operatorname{Ker} \lambda^{\prime}\right): W(\lambda)\right|$.

Proof. Let $w \in W$. Suppose that $\lambda^{w}$, considered as a linear character of the diagonal subgroup of $G$, is the restriction of a linear character of $P_{J}$. Then $\left(\lambda^{\prime}\right)^{w}$ is the restriction to $H$ of a linear character of $P_{0}$, and so $H \cap P_{0}^{\prime} \leq \operatorname{Ker}\left(\left(\lambda^{\prime}\right)^{w}\right)$, and also $H \cap P_{0}^{\prime} \leq \operatorname{Ker} \lambda^{\prime}$. Since $H /\left(H \cap P_{0}^{\prime}\right)$ is cyclic, and since $\operatorname{Ker} \lambda^{\prime}$ and $\operatorname{Ker}\left(\lambda^{\prime}\right)^{w}=\left(\operatorname{Ker} \lambda^{\prime}\right)^{w}$ have the same index in $H$, we have $\operatorname{Ker} \lambda^{\prime}=\left(\operatorname{Ker} \lambda^{\prime}\right)^{w}$. Hence $w$ normalizes $\operatorname{Ker} \lambda^{\prime}$. The desired inequality follows.

LEMMA 2.13. $N_{W}\left(\operatorname{Ker} \lambda^{\prime}\right) \leq N_{W}\left(\widehat{C}\left(\lambda^{\prime}\right)\right)$.

Proof. Since $H \cap P_{0}^{\prime} \leq \operatorname{Ker} \lambda^{\prime}$ and $H / H \cap P_{0}^{\prime}$ is cyclic, $H / \operatorname{Ker} \lambda^{\prime}$ has an abelian automorphism group. Hence $\left(N_{W}\left(\operatorname{Ker} \lambda^{\prime}\right)\right)^{\prime} \leq W\left(\lambda^{\prime}\right)$. Clearly $W\left(\lambda^{\prime}\right) \leq N_{W}\left(\operatorname{Ker} \lambda^{\prime}\right)$. Thus $W\left(\lambda^{\prime}\right)$ is normal in $N_{W}\left(\operatorname{Ker} \lambda^{\prime}\right)$. Then $N_{W}\left(\operatorname{Ker} \lambda^{\prime}\right)$ permutes the reflections in $W(\lambda)^{\prime}$ and so normalizes $\widehat{C}\left(\lambda^{\prime}\right)$.

LEMMA 2.14. $C(\lambda)=C\left(\lambda^{\prime}\right)$.

Proof. By Remark 2.3, $C(\lambda)$ is the reflection group with root system $\Gamma=\left\{a \in \Phi: q_{a}(\lambda) \neq 1\right\}$, and $C\left(\lambda^{\prime}\right)$ is the reflection group with root system $\Gamma^{\prime}=\left\{a \in \Phi: q_{a}\left(\lambda^{\prime}\right) \neq 1\right\}$. By the proof of Lemma 2.4, $q_{a}(\lambda)$ is determined by the value of $\lambda$ on elements of $H$. Hence $q_{a}(\lambda)=q_{a}\left(\lambda^{\prime}\right)$ for all $a \in \Phi$. Thus $\Gamma=\Gamma^{\prime}$ and $C(\lambda)=C\left(\lambda^{\prime}\right)$.

LeMMA 2.15. $n(\lambda) \leq\left|N_{W}\left(\widehat{C}\left(\lambda^{\prime}\right)\right): \widehat{C}\left(\lambda^{\prime}\right)\right|\left|\widehat{C}\left(\lambda^{\prime}\right): C\left(\lambda^{\prime}\right)\right|$.

Proof. By Lemmas 2.12 and 2.13, $n(\lambda) \leq\left|N_{W}\left(\widehat{C}\left(\lambda^{\prime}\right)\right): W(\lambda)\right|$. Hence $n(\lambda) \leq\left|N_{W}\left(\widehat{C}\left(\lambda^{\prime}\right)\right): \widehat{C}\left(\lambda^{\prime}\right)\right|\left|\widehat{C}\left(\lambda^{\prime}\right): C(\lambda)\right|$. Now Lemma 2.14 yields the desired result.

LEMMA 2.16. Let $W_{J}$ be a maximal parabolic subgroup of an irreducible Weyl group $W$. Let $W_{1}$ be a reflection subgroup of $W$ which contains $W_{J}$. Then $\left|N_{W}\left(W_{1}\right): W_{1}\right| \leq 72$. 
Proof. We follow Carter [6]. Let $\Phi_{1}=\left\{a \in \Phi: w_{a} \in W_{1}\right\}$. Then $\Phi_{1}$ is a root system with Weyl group $W_{1}$. Note that $\Phi_{J} \leq \Phi_{1}$. Let $V_{1}$ be the vector space spanned by $\Phi_{1}$. The roots in $\Phi$ orthogonal to $\Phi_{1}$ form a subsystem $\Phi_{2}$ with Weyl group $W_{2}$. Clearly $W_{2}$ has order 1 or 2 in our situation. By [6, Proposition 28], $N_{W}\left(W_{1}\right) /\left(W_{1} \times W_{2}\right)$ is isomorphic to a group of symmetries of $\Delta_{1}$, the Dynkin diagram of $\Phi_{1}$.

If $W_{1}>W_{J}$, then $\Phi_{1}$ is obtained by deleting a node from the extended Dynkin diagram of $\Phi$, or is the dual of the diagram obtained by deleting a node from the extended Dynkin diagram of the root system dual to $\Phi$. See [6, p. 8]. An easy case-by-case check shows that the largest value of $\left|\operatorname{Aut}\left(\Delta_{1}\right)\right|\left|W_{2}\right|$ is 72 , which occurs when the middle node is deleted from the extended Dynkin diagram of $D_{8}$.

THEOREM 2.17. $n(\lambda) \leq 576$.

Proof. By Lemma 2.16, $\left|N_{W}\left(\widehat{C}\left(\lambda^{\prime}\right)\right): \widehat{C}\left(\lambda^{\prime}\right)\right| \leq 72$. Since $\widehat{C}\left(\lambda^{\prime}\right)$ normalizes $C\left(\lambda^{\prime}\right)$ and $\widehat{C}\left(\lambda^{\prime}\right) / C\left(\lambda^{\prime}\right)$ is an elementary abelian 2-group, the proof of Lemma 2.16 yields $\left|\widehat{C}\left(\lambda^{\prime}\right): C\left(\lambda^{\prime}\right)\right| \leq 8$. Lemma 2.15 then gives the desired conclusion.

3. Estimating $\chi_{3}$. Let $\left(G, x, L_{J}\right)$ be an admissible triple (see Def. 1.8) with rank $(G) \geq 2$. Let $\theta \in \operatorname{Irr}\left(U_{J}\right)$ be invariant under $L_{J}^{\prime}$. Let $\chi \in \operatorname{Irr}\left(P_{J}\right)$ lie over $\theta$. (In the situation of the introduction to this paper, $\chi$ plays the role of an irreducible constituent of $\chi_{3}$.) In this section, we establish the bound $|\chi(x) / \chi(1)| \leq 1 / q$.

Relying heavily on results from [2], we show that $\left[U_{J}^{\prime}, U_{J}\right] \leq \operatorname{Ker} \theta$, that $U_{J} / \operatorname{Ker} \theta$ is extraspecial, and that $x$ preserves a $\operatorname{GF}(q)$-bilinear symplectic form on $U_{J} / U_{J}^{\prime}$. We then restrict to $\langle x\rangle U_{J}$ and use standard Hall-Higman type results to obtain the desired bound.

For an admissible triple $\left(G, x, L_{J}\right)$, let $\widehat{H}$ denote the diagonal subgroup of $G$. Let $G^{\prime}=\bar{G}_{\sigma}$ where $\bar{G}$ is a simple algebraic group. Here $\sigma=q \tau$, where $q$ is the $q$ th power map on $\overline{\mathrm{GF}(p)}$ extended to a Frobenius morphism of $\bar{G}$ and $\tau$ is a possibly trivial graph automorphism of $\bar{G}$.

Let $P=P_{J} \cap G^{\prime}, L=L_{J} \cap G^{\prime}$, and $H=\widehat{H} \cap G^{\prime}$. Let $L_{0}=L^{\prime}=L_{J}^{\prime}$ and let $H_{0}=H \cap L_{0}=\widehat{H} \cap L_{0}$. This notation differs from that of $\S 2$.

Let $\Sigma$ be the root system of $\bar{G}$, and let $\Pi$ be a fundamental system for $\Sigma$. Choose $K \leq \Pi$, abusing notation, so that $P=\left(\bar{P}_{K}\right)_{\sigma}$, where 
$\bar{P}_{K}$ is the standard parabolic of $\bar{G}$ corresponding to $K$. Thus $K^{\prime}=$ $\Pi-K$ will consist of a single $\tau$-orbit of fundamental roots. The unipotent radical $\bar{U}_{K}$ of $\bar{P}_{K}$ is the product of the root groups $\bar{U}_{\beta}$ for $\beta \in \Sigma^{+}-\Sigma_{K}$.

We differ slightly from [2], where instead of $\bar{P}_{K}$, the authors work with the "opposite" parabolic generated by $\bar{L}_{K}$ and the negative root groups in $\Sigma-\Sigma_{K}$. By [2, p. 561], passing from $\bar{P}_{K}$ and $\left(\bar{P}_{K}\right)_{\sigma}$ to their opposites merely replaces the modules $W_{S}$ defined below by their duals, which is harmless for our purposes. We also remark that the results of [2] apply to all parabolic subgroups, not just the maximal parabolics considered here.

For $\beta \in \Sigma^{+}-\Sigma_{K}$, write $\beta=\beta_{K}+\beta_{K^{\prime}}$, where $\beta_{K}$ is a linear combination of fundamental roots in $K$, and similarly for $\beta_{K^{\prime}}$. Write $\beta_{K^{\prime}}=d_{1} \alpha_{1}+\cdots+d_{i} \alpha_{i}+\cdots$, where $\alpha_{i}$ ranges over $K^{\prime}$. Following [2, p. 3], define the shape of $\beta$ to be $\beta_{K^{\prime}}$ and the level of $\beta$ to be the sum of the $d_{i}$ above.

Let $\bar{U}(i)=\prod \bar{U}_{\beta}$, the product over all $\beta \in \Sigma^{+}-\Sigma_{K}$ with level $(\beta) \geq i$. Let $U(i)=\bar{U}(i)_{\sigma}$. Then $U(i)$ is the product of the corresponding root groups of $G^{\prime}=\bar{G}_{\sigma}$. By [2, Lemma 4 and Lemma 6], $U(1)>U(2)>\cdots$ is the descending central series of $U_{J}=\left(\bar{U}_{K}\right)_{\sigma}$.

Let $M(i)=U(i) / U(i+1)$. By [2, Lemma 5], $M(i)$ is $L$-isomorphic to $(\bar{U}(i) / \bar{U}(i+1))_{\sigma}$. By [2, Theorem 2a and Theorem 3], $M(i)$ has a direct decomposition as a product of $P$-chief factors $W_{S}$, which we will describe below. Each $W_{S}$ is an irreducible $\operatorname{GF}\left(q^{c}\right)[L]$-module, where $c$ is the number of shapes in the $\tau$-orbit of the shape $S$.

The results above need not hold without our assumption that $\bar{G}$ is not "special".

To describe $W_{S}$, we need more notation. For $G$ untwisted and $S$ a shape on level $i$, define $V_{S}$ to be the image in $M(i)$ of the product of all root subgroups $U_{\beta}$, for $\beta$ of shape $S$. Define $\bar{V}_{S}$ similarly for $\bar{G}$.

If $G$ is untwisted put $W_{S}=V_{S}$. If $G$ is twisted and $S^{\tau}=S$, put $W_{S}=\left(\bar{V}_{S}\right)_{\sigma}$. If $G$ is twisted and $S^{\tau} \neq S$ let $W_{S}$ equal $\left(\bar{V}_{S} \oplus \bar{V}_{S}^{\sigma}\right)_{\sigma}=$ $\left(\bar{V}_{S} \oplus \bar{V}_{S^{\tau}}\right)_{\sigma}$ when $|\tau|=2$, or the obvious analog when $|\tau|=3$. By [2, Theorem $2 \mathrm{~b}$ and Lemma 7], $W_{S}$ is an irreducible $\mathrm{GF}\left(q^{c}\right)[L]$-module. There is one module $W_{S}$ for each $\tau$-orbit of shapes in $\Sigma^{+}-\Sigma_{K}$.

By [2, Theorem 2d and Lemma 7], $W_{S}$ remains irreducible as a $\mathrm{GF}\left(q^{c}\right)\left[L_{0}\right]$-module. Finally, the $W_{S}$ are also $P_{J}$-chief factors, since $P_{J}=P \widehat{H}$ and the $W_{S}$ are invariant under diagonal automorphisms; see $[2$, p. 552]. 
In Lemma 3.1 below, we give a criterion for $W_{S}$ to be centralized by $L_{0}$. In Lemmas 3.2 and 3.3, we locate the trivial $L_{0}$-composition factors in $U_{J}$.

LEMMA 3.1. $L_{0}$ centralizes $W_{S}$ iff there is only one root in $\Sigma^{+}-\Sigma_{K}$ of shape $S$.

Proof. First suppose $\tau=1$. Then, since $W_{S}=V_{S}$ is an irreducible $\mathrm{GF}(q)\left[L_{0}\right]$-module, $L_{0}$ centralizes $V_{S}$ iff $V_{S}$ has $\mathrm{GF}(q)$-dimension 1 , iff there is only one root in $S$.

Next suppose $|\tau|=2$. Then $\tau$ induces a linear transformation on $\overline{M(i)}=\bar{U}(i) / \bar{U}(i+1)$ which permutes the images of the root groups in $\bar{G}$ on level $i$. Suppose first that $\left(\bar{V}_{S}\right)^{\sigma}=\bar{V}_{S}$. Then $S=S^{\tau}$, the roots of shape $S$ comprise a union of $\tau$-orbits, and $\left(\bar{V}_{S}\right)_{\sigma}=W_{S}$ is the direct sum of the $\sigma$-fixed point spaces for each of these $\tau$-orbits. For each such $\tau$-orbit, the $\sigma$-fixed point space is the image in $M(i)$ of a root group of the twisted group $\bar{G}_{\sigma}=G^{\prime}$. This image has cardinality $q$ if the $\tau$-orbit consists of one root, and cardinality $q^{2}$ if the $\tau$-orbit consists of two roots. If $L_{0}$ centralizes $\left(\bar{V}_{S}\right)_{\sigma}=W_{S}$, then $\left(\bar{V}_{S}\right)_{\sigma}$ has dimension 1 over $\operatorname{GF}(q)$. Hence $S$ consists of only one $\tau$-orbit, which in turn consists of only one root. Conversely, if $S$ consists of only one root, then $\left(\bar{V}_{S}\right)_{\sigma}$ has cardinality $q$, and so is centralized by $L_{0}$.

Next suppose $|\tau|=2$ and $\left(\bar{V}_{S}\right)^{\sigma} \neq \bar{V}_{S}$. Again $W_{S}=\left(\bar{V}_{S} \oplus \bar{V}_{S}^{\sigma}\right)_{\sigma}$ is the direct sum of the $\sigma$-fixed point spaces for the $\tau$-orbits of roots in $S \cup S^{\tau}$. Since $S$ and $S^{\tau}$ are disjoint, each such $\tau$-orbit consists of two roots, and so its $\sigma$-fixed point space in $M(i)$ has cardinality $q^{2}$, as above. If $L_{0}$ acts trivially on $W_{S}$, then $\operatorname{GF}\left(q^{2}\right)\left[L_{0}\right]$-irreduciblity forces $W_{S}$ to have dimension 1 over $\operatorname{GF}\left(q^{2}\right)$. Hence $S \cup S^{\tau}$ contains only one $\tau$-orbit of roots, and so $S$ contains only one root. The converse is clear.

Finally, the case $|\tau|=3$ is entirely similar to $|\tau|=2$.

LemMA 3.2. Let $S$ be a shape in $\Sigma^{+}-\Sigma_{K}$ with level $(S)=1$. Then $S$ contains more than one root.

Proof. First suppose $G$ is untwisted. Let $K^{\prime}=\{\alpha\}$. All roots on level 1 have the same shape. To show that this shape contains a root distinct from $\alpha$, we need only show that for some fundamental root $\beta \neq \alpha$, that $\alpha+k \beta$ is a root for some positive integer $k$. Root chain considerations [7, p. 37] show that this is the case whenever $\beta$ is not orthogonal to $\alpha$. 
Now suppose $G$ is twisted. If $\left|K^{\prime}\right|=1$, then the unique shape on level 1 contains more than one root, as above. We then assume $\left|K^{\prime}\right|>1$. The shape $S$ consists of all roots of the form $\alpha+\sum_{i \in K} c_{i} \alpha_{i}$, where $\alpha$ is a fixed fundamental root in $K^{\prime}$. An easy case-by-case check shows that there is always a fundamental root $\alpha_{i} \in K$ which is not orthogonal to $\alpha$. (Recall that $G$ has rank at least 2 as a twisted group.) Hence we get two roots of shape $S$ as above.

LEMMA 3.3. Let $S$ be a shape in $\Sigma^{+}-\Sigma_{K}$ which contains only one root. Then level $(S)=2$ and $L_{0}$ centralizes $M(2)$. The commutator form from $M(1) \times M(1)$ to $M(2)$ is nondegenerate, $\mathrm{GF}(q)$-bilinear, and $L_{0}$-invariant.

Proof. First we claim that level $(S)=2$. Suppose $G$ is untwisted. If $G$ is a classical group, there are at most two levels of roots in $\Sigma^{+}-\Sigma_{K}$, and so our claim follows from Lemma 3.2. For the exceptional groups, we merely check the lists of positive roots in [4] or [19]. We find that for $F_{4}, E_{6}, E_{7}$, and $E_{8}$, there is exactly one maximal subset $K$ of $\Pi$ for which $\Sigma^{+}-\Sigma_{K}$ has a shape (i.e. level) consisting of just one root, and this always occurs on level 2 . For such $K$, there are only two levels of roots in $\Sigma^{+}-\Sigma_{K}$. For type $G_{2}$, both possibilities for $K$ have a unique root (only) on level 2, and there are two or three levels of roots in $\Sigma^{+}-\Sigma_{K}$.

Next suppose $G$ is twisted. If $\left|K^{\prime}\right|=1$, then a shape in $\Sigma^{+}-\Sigma_{K}$ is the same thing as a level, and the claim follows as in the preceding paragraph. Hence we assume $\left|K^{\prime}\right|>1$. For types ${ }^{2} A_{n}$ and ${ }^{2} D_{n}$, the highest level in $\Sigma^{+}-\Sigma_{K}$ is 2 . For type ${ }^{3} D_{4}$, with $K=\left\{\alpha_{2}\right\}$, the fundamental root fixed by $\tau$, there are three levels. Level 2 consists of single $\tau$-orbit of shapes, each containing only one root. Level 3 consists of one $\tau$-invariant shape, which contains two roots. For type ${ }^{2} E_{6}$, there are only two levels when $K^{\prime}=\left\{\alpha_{1}, \alpha_{6}\right\}$, the outer pair of nodes exchanged by $\tau$. When $K^{\prime}=\left\{\alpha_{3}, \alpha_{5}\right\}$, the inner pair of nodes exchanged by $\tau$, there are four levels. The shapes of level at least 2 for these sets $K^{\prime}$ are $\alpha_{1}+\alpha_{6}, \alpha_{3}+\alpha_{5}, 2 \alpha_{3}+\alpha_{5}, \alpha_{3}+2 \alpha_{5}$, and $2 \alpha_{3}+2 \alpha_{5}$. All of these shapes contain at least two roots. This proves our first claim.

We next claim that $L_{0}$ centralizes $M(2)$. By Lemma 3.1, it suffices to show that there is only one $\tau$-orbit of shapes on level 2 , since $M(2)$ would then be a trivial $\operatorname{GF}\left(q^{c}\right)\left[L_{0}\right]$-module of the form $W_{S}$. If $G$ is untwisted, or if $G$ is twisted with $\left|K^{\prime}\right|=1$, this is clear since shape and level are then the same thing. If $G$ is twisted with $\left|K^{\prime}\right|>1$, 
then our assumption that $\Sigma^{+}-\Sigma_{K}$ has a shape containing only one root implies that $G$ is not of type ${ }^{2} E_{6}$. If $G$ is of type ${ }^{3} D_{4}$, then there is only one $\tau$-orbit of shapes on level 2 as mentioned above. If $G$ is of type ${ }^{2} D_{n}$, then $\left|K^{\prime}\right|>1$ implies that $K^{\prime}=\left\{\alpha_{n-1}, \alpha_{n}\right\}$, the pair of fundamental roots exchanged by $\tau$. The only shape on level 2 is then $\alpha_{n-1}+\alpha_{n}$, which contains at least two roots. Our claim is therefore vacuously satisfied for type ${ }^{2} D_{n}$. Note, by the way, that the description of the positive roots of $D_{n}$ on $[4$, p. 256] is incorrect. If $G$ is of type ${ }^{2} A_{n}$, then we get a one-root shape only when $K^{\prime}=\left\{\alpha_{1}, \alpha_{n}\right\}$, the outermost pair of nodes exchanged by $\tau$. Here there is only one shape on level 2 , namely $\alpha_{1}+\alpha_{n}$. This proves our second claim.

We now claim that there is only one $\tau$-orbit of shapes on level 1 . As in the preceding paragraph, this is clear when $G$ is untwisted or when $\left|K^{\prime}\right|=1$. The only other cases we have to consider are ${ }^{3} D_{4}$ with $K=\left\{\alpha_{2}\right\}$ and ${ }^{2} A_{n}$ with $K^{\prime}=\left\{\alpha_{1}, \alpha_{n}\right\}$. In both cases we see that there is a unique $\tau$-orbit of shapes on level 1 . This proves our claim. It follows that $M(1)$ is a single $P$-chief factor.

Since $U(1)^{\prime}=U(2)$ and $[U(1), U(2)]=U(3)$, there is a welldefined commutator form from $M(1) \times M(1)$ to $M(2)$. Since $L_{0}$ centralizes $M(2)$, this form is $L_{0}$-invariant. Since $M(1)$ is a $P$-chief factor, $Z(U(1) / U(3))=U(2) / U(3)=M(2)$. Hence the commutator form is nondegenerate.

It remains to show that the commutator form is $\mathrm{GF}(q)$-bilinear. If $G$ is untwisted, the $\operatorname{GF}(q)$-structure on each $M(i)$ is determined by $s x_{\beta}(t)=x_{\beta}(s t)$, where $s$ and $t$ are scalars in $\operatorname{GF}(q)$ and $x_{\beta}(t)$ is a root element for $\beta \in \Sigma^{+}-\Sigma_{K}$; see [2, p. 554]. For $G$ twisted, we have $s\left(x_{\beta}(t) x_{\beta^{\tau}}\left(t^{q}\right)\right)=x_{\beta}(s t) x_{\beta^{\tau}}\left(s t^{q}\right)$ when $|\tau|=2$ and $s \in \operatorname{GF}(q)$, $t \in \operatorname{GF}\left(q^{2}\right)$, etc. The Chevalley commutator formula now implies that the commutator form is $\mathrm{GF}(q)$-bilinear. This completes the proof of Lemma 3.3.

The key to our next result is the Glauberman character correspondence $[15,13.1]$, which says that when a solvable group $S$ acts coprimely on a group $G$, there is a one-to-one correspondence between the set of $S$-invariant irreducible characters of $G$ and the irreducible characters of $C_{G}(S)$.

Proposition 3.4. Let $1 \neq \theta \in \operatorname{Irr}\left(U_{J}\right)$ be $L_{0}$-invariant. Then $U(3) \leq \operatorname{Ker} \theta$ and $U_{J} / \operatorname{Ker} \theta$ is extraspecial. Also $L_{0}$ centralizes $M(2)$. 
Proof. Since $H_{0}$ fixes $\theta,[15,13.24]$ implies that $H_{0}$ fixes a nonidentity conjugacy class of $U_{J}$. By $[15,13.10], H_{0}$ fixes a nonidentity element $u \in U_{J}$. Writing $u$ as a unique product of elements from root groups, we see that $H_{0}$ centralizes a nonidentity element $v$ of some root group in $U_{J}$.

We claim that $C_{U_{J}}\left(H_{0}\right)$ is a root group of order $q^{3}$ when $G$ is of type ${ }^{3} D_{4}$, and is a root group of order $q$ in all other cases, and also $C_{U_{J}}\left(H_{0}\right)=C_{U_{J}}\left(L_{0}\right)$.

First suppose $G$ is untwisted. Then $v=x_{\gamma}(t)$, for some $\gamma \in$ $\Sigma^{+}-\Sigma_{K}$ and $t \in \operatorname{GF}(q)^{*}$. For $\beta \in \Pi_{J}$ and $s \in \operatorname{GF}(q)^{*}$, there is an element $h_{\beta}(s) \in H_{0}$ which conjugates $v$ into $x_{\gamma}\left(s^{A_{\beta \gamma}} t\right)$; see [7, p. 194]. Here $A_{\beta \gamma}$ is the Cartan integer associated with $\beta$ and $\gamma$ ([7, p. 38]).

Since $H_{0}$ centralizes $v$, it follows that $s^{A_{\beta \gamma}}=1$ for all $s \in \operatorname{GF}\left(q^{*}\right)$. Since $\left|A_{\beta \gamma}\right| \leq 3$, with equality only when $\Sigma$ is $G_{2}$, this implies that $A_{\beta \gamma}=0$ when $q \geq 5$. The same is true when $q=4$, since we are excluding the "special" group $G_{2}(4)$.

Hence $\gamma$ is orthogonal to $\Sigma_{J}$. It follows that $\left[U_{\beta}, U_{\gamma}\right]=1$ for all $\beta \in \Sigma_{J}$, and so $L_{0}$ centralizes $U_{\gamma}$. The maximality of $J$ implies that $\gamma$ must be the unique positive root orthogonal to $\Sigma_{J}$. It follows that $C_{U_{J}}\left(H_{0}\right)=C_{U_{J}}\left(L_{0}\right)=U_{\gamma}$, which has order $q$.

Now suppose that $G$ is twisted. Viewing $G^{\prime}$ as a subgroup of an untwisted Chevalley group $\widehat{G}$ over $\operatorname{GF}\left(q^{|\tau|}\right)$, write $v$ as a product of root elements of $\widehat{G}$ as in [7, 13.6.3]. (Since we are only interested in computing $C_{U_{J}}\left(H_{0}\right)$, it doesn't matter that $G^{\prime}$ is simply connected, while adjoint groups are considered in [7].) Then $v=x_{\gamma}(t) y$, where $\gamma \in \Sigma^{+}-\Sigma_{K}, t \neq 0$, and $y$ is a product of elements in other root subgroups of $\widehat{G}$ which belong to the $\tau$-equivalence class of $\gamma$. Since $H_{0}$ normalizes each root subgroup of $\widehat{G}$, we see that $H_{0}$ centralizes $x_{\gamma}(t)$ in $\widehat{G}$. By [7, p. 239-41], the generators of $H_{0}$ have the form $h_{\beta}(s) \quad\left(s \in \mathrm{GF}(q)^{*}\right)$, or $h_{\beta}(s) h_{\beta^{\tau}}\left(s^{q}\right) \quad\left(s \in \mathrm{GF}\left(q^{2}\right)^{*}\right)$, or the analog for $s \in \mathrm{GF}\left(q^{3}\right)^{*}$, where in all cases $\beta \in \Pi_{K}$. It follows that, respectively, $s^{A_{\beta \gamma}}=1$ for all $s \in \mathrm{GF}(q)^{*}, s^{A_{\beta \gamma}+q A_{\beta^{\tau} \gamma}}=1$ for all $s \in \operatorname{GF}\left(q^{2}\right)^{*}$, or the analogous equation holds for all $s \in \mathrm{GF}\left(q^{3}\right)^{*}$. This implies that $\gamma$ is orthogonal to $\Pi_{K}$, and hence to $\Sigma_{K}$ when $q \geq 4$.

It follows as above that every root subgroup $U_{\beta}$ of $\widehat{G}$, for $\beta \in \Sigma_{K}$, centralizes $U_{\gamma}$ and the root groups of $\widehat{G}$ in the $\tau$-equivalence class of $U_{\gamma}$. Hence $L_{0}$ centralizes the root subgroup of $G$ which corresponds to the $\tau$-equivalence class of $\gamma$. The preceding argument shows that any root group in $U_{J}$ centralized by $H_{0}$ is also centralized by $L_{0}$. 
We claim that, as in the untwisted case, there is only one root group in $U_{J}$ centralized by $H_{0}$. When $\left|K^{\prime}\right|=1$, this is clear because $\gamma$, in the preceding paragraph, must be the unique positive root orthogonal to the maximal parabolic subsystem $\Sigma_{K}$. Since $\gamma$ is then $\tau$-invariant, $\left|C_{U_{J}}\left(H_{0}\right)\right|=q$ when $\left|K^{\prime}\right|=1$.

Now suppose $\left|K^{\prime}\right|>1$. Since $L_{0}$ centralizes a root group in $U_{J}, L_{0}$ centralizes the image in some $M(i)$ of this root group. This root group image is then invariant under $L=L_{0} H$. Hence $L_{0}$ centralizes some $P$-chief factor in $U_{J}$, so $L_{0}$ centralizes $W_{S}$ for some shape $S$. By Lemma 3.1 , there is only one root in $\Sigma^{+}-\Sigma_{K}$ of shape $S$. Thus $S$ satisfies the hypotheses of Lemma 3.3. The proof of Lemma 3.3 shows that, since $\left|K^{\prime}\right|>1, G$ must be of type ${ }^{2} A_{n}$ or ${ }^{3} D_{4}$ with $K^{\prime}=\left\{\alpha_{1}, \alpha_{n}\right\}$ or $\left\{\alpha_{1}, \alpha_{3}, \alpha_{4}\right\}$ respectively. In both cases, $L_{0}$ acts trivially on $M(2)$, which is isomorphic to a root subgroup of $G$, namely $U_{\alpha_{1}+\cdots+\alpha_{n}}$ or $\left(\bar{U}_{\alpha_{1}+\alpha_{2}+\alpha_{3}} \bar{U}_{\alpha_{1}+\alpha_{2}+\alpha_{4}} \bar{U}_{\alpha_{2}+\alpha_{3}+\alpha_{4}}\right)_{\sigma}$. Moreover, $L_{0}$ has no other trivial composition factors in $U_{J}$. Since any root subgroup centralized by $H_{0}$ is also centralized by $L_{0}$, as remarked above, it follows that $C_{U_{J}}\left(H_{0}\right)$ is a single root subgroup of $G$. This root subgroup has order $q$ when $G$ is of type ${ }^{2} A_{n}$ and order $q^{3}$ when $G$ is of type ${ }^{3} D_{4}$.

Thus we have proved the claim in the second paragraph of this proof.

Having just observed that $C_{U_{J}}\left(H_{0}\right)$ maps isomorphically onto $M(2)$ when $G$ is twisted and $\left|K^{\prime}\right|>1$, we claim the same is true when $G$ is untwisted or when $\left|K^{\prime}\right|=1$. Under these hypotheses, shape and level are the same thing, and each shape is $\tau$-invariant. It follows that each $M(i)$ is an irreducible $\operatorname{GF}(q)\left[L_{0}\right]$-module. If $L_{0}$ centralizes a vector in $M(i)$, then $L_{0}$ centralizes $M(i)$ and, by Lemmas 3.1 and 3.3, we have $i=2$ and the unique shape on level 2 contains only one root. Hence if $L_{0}$ centralizes a root subgroup $\left(\bar{U}_{\gamma}\right)_{\sigma}$ in $U_{J}$, then $\gamma$ is the unique root in $\Sigma^{+}-\Sigma_{K}$ on level 2 . Hence $C_{U_{J}}\left(H_{0}\right)=\left(\bar{U}_{\gamma}\right)_{\sigma}$ maps isomorphically onto $M(2)$.

Thus in all cases $C_{U_{J}}\left(H_{0}\right)=C_{U_{J}}\left(L_{0}\right)$ maps isomorphically onto $M(2)$. In particular, $L_{0}$ centralizes $M(2)$.

Let $1 \neq \lambda$ be a linear character of $M(2)$. View $\lambda$ as an irreducible character of $U(2)$.

Suppose first that $G$ is not of type ${ }^{3} D_{4}$. Then the nondegeneracy and the $\mathrm{GF}(q)$-bilinearity of the commutator form from $M(1) \times M(1)$ to $M(2)$ and the fact that $|M(2)|=q$ implies that $[v, M(1)]=M(2)$ for any nonzero vector $v \in M(1)$. It follows that $Z\left(U_{J} / \operatorname{Ker} \lambda\right)=$ $U(2) / \operatorname{Ker} \lambda$. Hence $U_{J} / \operatorname{Ker} \lambda$ is extraspecial. There is a unique 
character $\theta_{\lambda} \in \operatorname{Irr}\left(U_{J} / \operatorname{Ker} \lambda\right)$ which lies over $\lambda$. Since $L_{0}$ centralizes $M(2), \theta_{\lambda}$ is an $L_{0}$-invariant character of $U_{J}$. Together with the principal character of $U_{J}$, this yields $|M(2)|=q$ distinct $L_{0}$-invariant irreducible characters of $U_{J}$. Since $|M(2)|=\left|C_{U_{J}}\left(H_{0}\right)\right|$ and since $C_{U_{J}}\left(H_{0}\right)$ is abelian, Glauberman's correspondence $[15,13.1]$ implies that there are no other $H_{0}$-invariant irreducible characters of $U_{J}$, and therefore no other $L_{0}$-invariant irreducible characters of $U_{J}$. Since $\operatorname{Ker} \theta_{\lambda}=\operatorname{Ker} \lambda, U_{J} / \operatorname{Ker} \theta_{\lambda}$ is extraspecial, as desired.

Next suppose $G$ has type ${ }^{3} D_{4}$. Let $Z=Z\left(U_{J} / \operatorname{Ker} \lambda\right)$. Since $L_{0}$ fixes $\lambda, Z$ and $\bar{Z}=Z /(U(2) / \operatorname{Ker} \lambda)$ are $L_{0}$-invariant. If $\bar{z} \in$ $\bar{Z} \leq M(1)$, and $c \in \mathrm{GF}(q)^{*}$, then $[c \bar{z}, M(1)]=[\bar{z}, c M(1)]=$ $[\bar{z}, M(1)] \leq \operatorname{Ker} \lambda$, and so $c \bar{z} \in \bar{Z}$. It follows that $\bar{Z}$ is a proper $\mathrm{GF}(q)\left[L_{0}\right]$-submodule of $M(1)$.

Since $C_{U_{J}}\left(H_{0}\right) \leq U(2), H_{0}$ has no fixed points on $M(1)$. Hence $C_{Z}\left(H_{0}\right)=U(2) / \operatorname{Ker} \lambda$, and so $Z=\left[H_{0}, Z\right] \times(U(2) / \operatorname{Ker} \lambda)$.

Let $\theta \in \operatorname{Irr}\left(U_{J} \mid \lambda\right)$ be $H_{0}$-invariant. View $\theta$ as a character in $\operatorname{Irr}\left(U_{J} / \operatorname{Ker} \lambda\right)$. Let $\lambda \times \mu \in \operatorname{Irr}(Z)=\operatorname{Irr}\left((U(2) / \operatorname{Ker} \lambda) \times\left[H_{0}, Z\right]\right)$ be the unique and linear irreducible consituent of $\theta_{Z}$. Since $\lambda \times \mu$ is $H_{0}$-invariant and $H_{0}$ has no fixed points on $\left[H_{0}, Z\right]$, we have $\mu=1$.

Let $E=\left(U_{J} / \operatorname{Ker} \lambda\right) /\left[H_{0}, Z\right]$. We claim that $E$ is extraspecial. Let $W=Z /\left[H_{0}, Z\right] \cong U(2) / \operatorname{Ker} \lambda$. Since $E / W$ is elementary abelian and $|W|=p$ it suffices to show that $Z(E)=W$. Let $y \in U_{J} / \operatorname{Ker} \lambda$ and suppose that $y\left[H_{0}, Z\right]$ belongs to $Z(E)$. Then $\left[y, U_{J} / \operatorname{Ker} \lambda\right] \leq\left[H_{0}, Z\right]$. Since $\left(U_{J} / \operatorname{Ker} \lambda\right)^{\prime}=U(2) / \operatorname{Ker} \lambda$, this implies that $y \in Z$, and so $y\left[H_{0}, Z\right] \in W$, as desired. Thus $E$ is extraspecial.

Let $\theta_{\lambda}$ be the unique character in $\operatorname{Irr}(E \mid \lambda)$. Since $\left[H_{0}, Z\right] \leq$ $\operatorname{Ker} \theta$, we have $\theta=\theta_{\lambda}$. In other words, $\theta_{\lambda}$ is the unique $H_{0}$-invariant character in $\operatorname{Irr}\left(U_{J} \mid \lambda\right)$. For each linear character $\lambda$ of $M(2)$, there is exactly one $H_{0}$-invariant character in $\operatorname{Irr}\left(U_{J} \mid \lambda\right)$. As above, the Glauberman correspondence shows that there are no other $H_{0}$ invariant irreducible characters of $U_{J}$. For $\lambda \neq 1, U_{J} / \operatorname{Ker} \theta_{\lambda}$ is extraspecial, as desired.

TheOREM 3.5. Let $\left(G, x, L_{J}\right)$ be an admissible triple with rank $(G) \geq 2$. Let $1 \neq \theta \in \operatorname{Irr}\left(U_{J}\right)$ be $L_{0}$-invariant. Let $\chi \in \operatorname{Irr}\left(P_{J} \mid \theta\right)$. Then $|\chi(x) / \chi(1)| \leq 1 / q$.

Proof. Let $I$ be the inertia group of $\theta$ in $P_{J}$. Since $P_{J}^{\prime}=L_{0} U_{J} \leq$ $I, I$ is normal in $P_{J}$. Hence if $x \notin I$, then $\chi(x)=0$. Therefore we assume that $x \in I$. 
Let $\chi=\psi^{P_{J}}$ with $\psi \in \operatorname{Irr}(I \mid \theta)$. Since $\chi(x)=\sum \psi\left(x^{t}\right)$, where $t$ ranges over a transversal to $I$ in $P_{J}$, it suffices to show that $\left|\psi\left(x^{t}\right) / \psi(1)\right| \leq 1 / q$ for each $t$. Fix $t$ and set $y=x^{t}$. Note that $y \in I$.

By Proposition 3.4, $\left.\theta\right|_{U(2)}$ has a unique and linear irreducible constituent $\lambda$. Since $y \in I, y$ fixes $\lambda$. The proof of Proposition 3.4 shows that $M(2)$, which has order $q$ or $q^{3}$, is a single $P$-chief factor $W_{S}$. Hence $M(2)$ is an irreducible $\operatorname{GF}(q)\left[L_{J}\right]$-module. Since $L_{0}$ centralizes $M(2)$ and $L_{J} / L_{0}$ is abelian, $C_{M(2)}(y)$ is a $\operatorname{GF}(q)\left[L_{J}\right]$ submodule of $M(2)$. Since $y$ fixes a linear character of $M(2), y$ fixes a nonzero vector in $M(2)$. Irreduciblity then forces $C_{M(2)}(y)=$ $M(2)$.

Let $E=U_{J} / \operatorname{Ker} \theta$. By Proposition 3.4 and its proof, $E$ is extraspecial and

$$
\begin{aligned}
E / Z(E) & =\left(U_{J} / \operatorname{Ker} \theta\right) /(U(2) \operatorname{Ker} \theta / \operatorname{Ker} \theta) \\
& \cong U_{J} / U(2) \operatorname{Ker} \theta
\end{aligned}
$$

By the proof of Proposition 3.4, $U(2) \operatorname{Ker} \theta=U(2)$ when $G$ is not of type ${ }^{3} D_{4}$. Whether $G$ has type ${ }^{3} D_{4}$ or not, $U(2) / \operatorname{Ker} \theta / U(2)$ is the image in $M(1)$ of $Z\left(U_{J} / \operatorname{Ker} \lambda\right)$, and is a proper (possibly zero) $\operatorname{GF}(q)\left[\left\langle L_{0}, y\right\rangle\right]$-submodule of $M(1)$. Hence $E / Z(E)$ can be identified with a $\operatorname{GF}(q)\left[\left\langle L_{0}, y\right\rangle\right]$-complement $M$ to $U(2) \operatorname{Ker} \theta / U(2)$ in $M(1)$.

By Lemmas 3.1 and $3.3, L_{0}$ centralizes no $P$-chief factor $W_{S} \leq$ $M(1)$. Since $W_{S}$ is an irreducible $\operatorname{GF}(q)[L]$-module, Clifford's Theorem implies that no $\operatorname{GF}(q)\left[L_{0}\right]$-submodule of $W_{S}$ is centralized by $L_{0}$. Hence $M(1)$ contains no trivial $\operatorname{GF}(q)\left[L_{0}\right]$-submodule. In particular, $L_{0}$ does not centralize $M$. Since $y$ centralizes no component of $L_{0}$, it follows that $y$ doesn't centralize $M$.

Since $y$ centralizes $M(2), y$ preserves the commutator form from $M(1) \times M(1)$ to $M(2)$, and so $y$ preserves the restriction of this form to $M \times M$. Since $E$ is extraspecial, this restricted form is nondegenerate.

Now $M$ is the orthogonal direct sum of $[M, y]$ and $C_{M}(y)$. Since $M$ is nondegenerate, so is $[M, y]$. Hence $[M, y]$ admits a nondegenerate $\operatorname{GF}(q)$-bilinear alternating form. Thus $|[M, y]| \geq q^{2}$ and so $|[E / Z(E), y]| \geq q^{2}$.

To show that $|\psi(y) / \psi(1)| \leq 1 / q$, it suffices to show that $|\nu(y) / \nu(1)|$ $\leq 1 / q$, where $\nu$ is an arbitrary irreducible constituent of $\left.\psi\right|_{\langle y\rangle U_{J}}$. 
Since $\psi_{U_{J}}$ is a multiple of $\theta$, we may view $\nu$ as a character in $\operatorname{Irr}(\langle y\rangle E \mid \theta)$.

Let $Z(E) \leq D \leq E$, with $D / Z(E)=[E / Z(E), y]$. Then $D$ is extraspecial, since $E / Z(E)$ is the orthogonal direct sum of $[E / Z(E), y]$ and $C_{E / Z(E)}(y)$.

Let $\omega$ be an arbitrary irreducible constituent of $\left.\nu\right|_{\langle y\rangle D}$. After multiplying $\omega$ by a linear character of $\langle y\rangle D / D$, we may assume that $C_{\langle y\rangle}(D) \leq \operatorname{Ker} \omega$. Then [14, V, 17.13] yields that $\omega(1)=$ $|[E / Z(E), y]|^{1 / 2}$ and $|\omega(y)|=1$. Applying this to each irreducible constituent of $\left.\nu\right|_{\langle y\rangle D}$, we obtain $|\nu(y) / \nu(1)| \leq|[E / Z(E), y]|^{-1 / 2}$. As shown above, the last quantity is at most $1 / q$. This completes the proof.

4. Main theorem. Let $G$ be a simple admissible group, $P_{J}$ a standard maximal parabolic of $G$, and $\chi \in \operatorname{Irr}(G)$. There exists an absolute constant $N$ such that $\chi_{P_{J}}$ has at most $N-1$ linear constituents, counting multiplicities. By Theorems 2.10 and 2.17, we may take $N=1+13 \cdot 576$. We now state our main theorem, which will be proved at the end of this section.

MAIN THEOREM. Let $(G, x)$ be a simple admissible pair, with $q \geq$ $3 N$, for $N$ as above. Let $\chi$ be a nonlinear irreducible character of $G$. Then $|\chi(x)| \leq(3 N / q) \chi(1)-N$.

LEMMA 4.1. Let $(G, x)$ be a simple admissible pair with rank $(G)=$ 1. Then $|\chi(x) / \chi(1)| \leq 2 /(q-1)$, for any nonlinear $\chi \in \operatorname{Irr}(G)$.

Proof. We have $G^{\prime}=\operatorname{SL}(2, q)$ or $\operatorname{SU}(3, q)$. By Lemma 1.10, or an entirely similar argument when $G^{\prime}=\operatorname{SU}(3, q)$, we see that it suffices to establish the inequality in the statement of this lemma for all noncentral semisimple elements of $\operatorname{SL}(2, q), \operatorname{GL}(2, q), \operatorname{SU}(3, q)$, and $\mathrm{U}(3, q)$.

For $\operatorname{SL}(2, q), \operatorname{GL}(2, q)$, and $\mathrm{U}(3, q)$, we can do this by checking character tables $([\mathbf{1 1}],[\mathbf{1 8}],[\mathbf{1 2}])$. It remains to consider $\mathrm{SU}(3, q)$, whose character table seems not to be available in the literature.

If $x \in G=\operatorname{SU}(3, q)$ is contained in no proper parabolic of $G$, then $C_{G}(x)$ is a torus of $G$, so $|\chi(x)| \leq\left|C_{G}(x)\right|^{1 / 2} \leq q+1$. By [16, p. 419], $\chi(1) \geq q^{2}-q$. It follows that $|\chi(x) / \chi(1)| \leq 2 /(q-1)$.

We may then assume that $x \in B=H U$, the standard Borel subgroup. Furthermore, we may assume $x \in H$. Let $\lambda$ be a linear constituent of $\chi_{B}$. Since $G$ is doubly transitive on the cosets of $B$, 
$\left(\lambda^{G}, \lambda^{G}\right) \leq\left(1_{B}^{G}, 1_{B}^{G}\right)=2$, and so $\left(\chi_{B}, \lambda\right)=1$. By Harish-Chandra's theorem $[10,70.15(\mathrm{~A})]$, all the linear constituents of $\chi_{B}$ are conjugate under the Weyl group of $G$, which has order 2 . Hence $\chi_{B}$ has at most two linear constituents, counting multiplicities.

Let $\theta$ be a nonlinear constituent of $\chi_{B}$ and let $\omega$ be an irreducible constituent of $\theta_{U}$. If $U^{\prime}=Z(U) \leq \operatorname{Ker} \omega$, then, since $H / Z(G)$ is fixed point free on $U / U^{\prime}$, we have $x \notin I_{B}(\omega) \triangleleft B$, and so $\theta(x)=0$.

If $Z(U) \not \operatorname{Ker} \omega$, then $\operatorname{Ker} \omega \leq Z(U)$ and $U / \operatorname{Ker} \omega$ is extraspecial. Since $I_{B}(\omega) \triangleleft B$, we may assume that $x \in I_{B}(\omega)$, since otherwise $\theta(x)=0$. Then $\left.\theta\right|_{\langle U, x\rangle}$ is a sum of extensions of $\omega$. Let $\zeta$ be one such extension. After multiplying $\zeta$ by a linear character, we may assume that $C_{\langle x\rangle}(U) \leq \operatorname{Ker} \zeta$. Now $\langle U, x\rangle / \operatorname{Ker} \zeta$ satisfies the hypotheses of [14, V, 17.13]. We conclude that $\zeta(1)=q$ and $|\zeta(x)|=1$. It follows that $|\theta(x) / \theta(1)| \leq 1 / q$.

Hence $|\chi(x)| \leq 2+(\chi(1)-2) / q$, assuming that $x$ lies in a proper parabolic of $G$. Since $\chi(1) \geq q^{2}-q$, we have $|\chi(x)| \leq 2 \chi(1) / q$, which completes the proof.

LEMMA 4.2. Let $\left(G, x, L_{J}\right)$ be an admissible triple with rank $(G) \geq$ 2 and $q \geq 3 N$. Let $\chi$ be a nonlinear irreducible character of $\left\langle x, L_{J}^{\prime}\right\rangle$ and assume that the Main Theorem holds for groups of smaller rank than $G$. Then $|\chi(x)| \leq(3 N / q) \chi(1)-N$.

Proof. Let $K$ be a component of $L_{J}$. Lemma 1.6 implies that $\langle x, K\rangle$ is a simple admissible group. In particular, if $L_{J}$ has only one component, then $\left(\left\langle x, L_{J}^{\prime}\right\rangle, x\right)$ is a simple admissible pair, and the inductive hypothesis yields the desired bound for $|\chi(x)|$.

We now assume that $L_{J}^{\prime}=K_{1} K_{2}$, the case of three components being entirely similar.

If $x \in L_{J}^{\prime}$, write $x=x_{1} x_{2}$, with $x_{1} \in K_{1}$ and $x_{2} \in K_{2}$. By the definition of admissible triple, $x_{i} \notin Z\left(K_{i}\right)$ for $i=1,2$, and so each $x_{i}$ has prime order modulo $Z\left(K_{i}\right)$. Then $\left(K_{i}, x_{i}\right)$ is a simple admissible pair for $i=1,2$. We may write $\chi(x)=\chi_{1}\left(x_{1}\right) \chi_{2}\left(x_{2}\right)$ with $\chi_{i} \in \operatorname{Irr}\left(K_{i}\right)$, for $i=1,2$. We assume both $\chi_{1}$ and $\chi_{2}$ are nonlinear, the other case being easier. The inductive hypothesis yields $\left|\chi_{i}\left(x_{i}\right)\right| \leq(3 N / q) \chi_{i}(1) N$ for $i=1,2$. Hence

$$
|\chi(x)| \leq\left((3 N / q) \chi_{1}(1)-N\right)\left((3 N / q) \chi_{2}(1)-N\right) .
$$

If $a$ and $b$ are real numbers, both greater than $N$, one checks that $(a-N)(b-N) \leq a b-N$. Hence $|\chi(x)| \leq(3 N / q)^{2} \chi(1)-N$. Since $q \geq 3 N$, the desired bound follows. 
We now assume that $x \notin L_{J}^{\prime}$. Let $y$ be the automorphism of $K_{1} K_{2}$ induced by $x$ and let $y_{i}$ be the restriction of $y$ to $\operatorname{Aut}\left(K_{i}\right)$ for $i=1,2$. Since $x$ centralizes no component of $L_{J}$, the orders of $y, y_{1}$ and $y_{2}$ are the same prime number $r$. We form the semidirect products $L=\langle y\rangle K_{1} K_{2}, L_{1}=\left\langle y_{1}\right\rangle K_{1}$, and $L_{2}=\left\langle y_{2}\right\rangle K_{2}$.

Our inductive hypothesis implies that $|\alpha(x)| \leq(3 N / q) \alpha(1)-N$ for any nonlinear irreducible character $\alpha$ of $\left\langle K_{i}, x\right\rangle$, since $\left(\left\langle K_{i}, x\right\rangle, x\right)$ is a simple admissible pair. Let $W_{i}=\left\langle w_{i}\right\rangle$ be a cyclic group of order $|x|$ and form the central product $\left\langle K_{i}, x\right\rangle * W_{i}$, where $w_{i}^{r}=$ $x^{r} \in Z\left(\left\langle K_{i}, x\right\rangle\right)$. Then $x w_{i}^{-1}$ has order $r$ and $\left\langle K_{i}, x\right\rangle * W_{i}=$ $\left\langle K_{i}, x w_{i}^{-1}\right\rangle W_{i}$. If $\alpha \in \operatorname{Irr}\left(\left\langle K_{i}, x\right\rangle\right)$, then $\alpha(x)=\alpha^{*}\left(x w_{i}^{-1}\right) \lambda\left(w_{i}\right)$, where $\alpha^{*} \in \operatorname{Irr}\left(\left\langle K_{i}, x w_{i}^{-1}\right\rangle\right)$ has the same degree as $\alpha$ and $\lambda$ is a linear character of $W_{i}$. Similarly if $\beta \in \operatorname{Irr}\left(\left\langle K_{i}, x w_{i}^{-1}\right\rangle\right)$, then $\beta\left(x w_{i}^{-1}\right)=\beta^{*}(x) \mu\left(w_{i}^{-1}\right)$, where $\beta^{*} \in \operatorname{Irr}\left(\left\langle K_{i}, x\right\rangle\right)$ has the same degree as $\beta$ and $\mu$ is a linear character of $W_{i}$. There is an obvious isomorphism from $L_{i}$ to $\left\langle x w_{i}^{-1}, K_{i}\right\rangle$ which takes $y_{i}$ to $x w_{i}^{-1}$. It follows that $\left|\gamma\left(y_{i}\right)\right| \leq(3 N / q) \gamma(1)-N$ for every nonlinear irreducible character $\gamma$ of $L_{i}$.

Let $Z=\langle z\rangle$ be a cyclic group of order $|x|$. Form the central product $\left(\langle x\rangle K_{1} K_{2}\right) * Z$, where $z^{r}=x^{r}$. Then $\left(\langle x\rangle K_{1} K_{2}\right) * Z=$ $\left(\left\langle x z^{-1}\right\rangle K_{1} K_{2}\right) * Z \cong L * Z$. The argument of the preceding paragraph shows that if $|\zeta(y)| \leq(3 N / q) \zeta(1)-N$ for every nonlinear irreducible character $\zeta$ of $L$, then the conclusion of the lemma holds.

We are therefore reduced to working with the split extensions $L, L_{1}$ and $L_{2}$.

Let $\pi: K_{1} \times K_{2} \rightarrow K_{1} K_{2}$ be the natural map. Then $y$ acts on $K_{1} \times K_{2}$, stabilizing (in fact centralizing) $\operatorname{Ker} \pi$. Let

$$
\eta:\langle y\rangle\left(K_{1} \times K_{2}\right) \rightarrow L_{1} \times L_{2}
$$

be the map sending $y^{k}\left(x_{1}, x_{2}\right)$ to $\left(y_{1}^{k} x_{1}, y_{2}^{k} x_{2}\right)$, for $k \in \mathbf{Z}, x_{i} \in K_{i}$, $i=1,2$. Then $\eta$ is an injective homomorphism. Since $Z\left(K_{i}\right) \leq$ $Z\left(\bar{K}_{i}\right)$ for an appropriate semisimple algebraic group $\bar{K}_{i}$ (see [8, 3.6.8]), it follows that $x$, which belongs to a reductive overgroup of $\bar{K}_{i}$, centralizes $Z\left(K_{i}\right)$. This implies that $\eta(\operatorname{Ker} \pi)$ is normal (in fact central) in $L_{1} \times L_{2}$, and so $\eta$ induces an embedding of $L \cong\langle y\rangle\left(K_{1} \times K_{2}\right) / \operatorname{Ker} \pi$ into $\left(L_{1} \times L_{2}\right) / \eta(\operatorname{Ker} \pi)$.

Let $\zeta \in \operatorname{Irr}(L)$ be nonlinear. Let $\chi_{1} \chi_{2}$ be an irreducible constituent of $\left.\zeta\right|_{K_{1} K_{2}}$ with $\chi_{i} \in \operatorname{Irr}\left(K_{i}\right), i=1,2$. If $\chi_{1} \chi_{2}$ is not $\langle y\rangle$-invariant, then $\zeta=\left(\chi_{1} \chi_{2}\right)^{L}$ and $\zeta(y)=0$. We may therefore assume that $\chi_{1} \chi_{2}$ is $\langle y\rangle$-invariant. It follows that $\left.\zeta\right|_{K_{1} K_{2}}=\chi_{1} \chi_{2}$ and $\chi_{i}$ is $\left\langle y_{i}\right\rangle$-invariant 
for $i=1,2$. Since $L_{i} / K_{i}$ is cyclic, $\chi_{i}$ extends to $\zeta_{i} \in \operatorname{Irr}\left(L_{i}\right)$ for $i=1,2$, and so $\chi_{1} \times \chi_{2} \in \operatorname{Irr}\left(K_{1} \times K_{2}\right)$ extends to $\zeta_{1} \times \zeta_{2} \in$ $\operatorname{Irr}\left(L_{1} \times L_{2}\right)$. Hence $\chi_{1} \chi_{2} \in \operatorname{Irr}\left(K_{1} K_{2}\right)=\operatorname{Irr}\left(\left(K_{1} \times K_{2}\right) / \eta(\operatorname{Ker} \pi)\right)$ extends to $\zeta_{1} \zeta_{2} \in \operatorname{Irr}\left(\left(L_{1} \times L_{2}\right) / \eta(\operatorname{Ker} \pi)\right)$.

Viewing $L$ as a subgroup of $\left(L_{1} \times L_{2}\right) / \eta(\operatorname{Ker} \pi)$, we see that $\chi_{1} \chi_{2} \in$ $\operatorname{Irr}\left(K_{1} K_{2}\right)$ is extendible to $\left(\zeta_{1} \zeta_{2}\right)_{L} \in \operatorname{Irr}(L)$. Hence $\zeta=\lambda\left(\zeta_{1} \zeta_{2}\right)_{L}$ where $\lambda$ is a linear character of $L / K_{1} K_{2}$. Hence $|\zeta(y)|=\left|\zeta_{1} \zeta_{2}(\eta(y))\right|$ $=\left|\zeta_{1}\left(y_{1}\right)\right|\left|\zeta_{2}\left(y_{2}\right)\right|$. As proved above, our inductive hypothesis implies that $\left|\zeta_{i}\left(y_{i}\right)\right| \leq(3 N / q) \zeta_{i}(1)-N$ if $\zeta_{i}$ is nonlinear. The desired bound for $|\zeta(y)|$ now follows as in the third paragraph of this proof. As remarked above, this completes the proof.

Lemma 4.3. Let $(G, x)$ be an admissible pair. Suppose $x$ centralizes no component of $G$. Let $\theta \in \operatorname{Irr}(G)$ with $1+\theta$ a (not necessarily faithful) doubly transitive permutation character of $G$. Assume $q>11$. Then $|\theta(x)| \leq(3 N / q) \theta(1)-2 N$.

Proof. By [5, p. 8], $S \leq G / \operatorname{Ker} \theta \leq \operatorname{Aut}(S)$ for a simple group $S$. Either $S$ has Lie rank 1 or $S$ is some $\operatorname{PSL}(n, q)$. Since $x$ centralizes no component of $G, x \notin \operatorname{Ker} \theta$. Set $\bar{G}=G / \operatorname{Ker} \theta$.

In the rank 1 case, since $q>11, \theta$ is the Steinberg character of $\bar{G}$. Hence $|\theta(x)| \leq \theta(1) / q \leq(3 N / q) \theta(1)-2 N$, since $\theta(1) \geq q$.

We now assume $S=\operatorname{PSL}(n, q)$ with $n \geq 3$. Then $\bar{G}$ is $\operatorname{PSL}(n, q)$ or $\langle y\rangle \operatorname{PSL}(n, q)$, where $y$ is a $p^{\prime}$-element of prime order. Since $y$ induces an inner times diagonal automorphism of $\operatorname{PSL}(n, q), \bar{G}$ is isomorphic to a subgroup of $\operatorname{PGL}(n, q)$. By [5, p. 8] $\bar{G}$ has two doubly transitive permutation representations, which are conjugate under the inverse transpose automorphism of $\operatorname{PGL}(n, q)$. Hence we may assume that $1+\theta$ is the permutation character for the action of $\bar{G}$ on the points of $\operatorname{PG}(n-1, q)$. Let $d_{1}, \ldots, d_{r}$ be the dimensions of the eigenspaces, if any, of a preimage of $\bar{x}$ in $\operatorname{GL}(n, q)$. Then $1+\theta(x)=0$ or $1+\theta(x)=\left(\left(q^{d_{1}}-1\right)+\cdots+\left(q^{d_{r}}-1\right)\right) /(q-1) \leq$ $\left(\left(q^{n-1}-1\right)+(q-1)\right) /(q-1)$. If $\theta(x) \neq-1$, then $0 \leq \theta(x) / \theta(1) \leq$ $(1+\theta(x)) /(1+\theta(1)) \leq\left(q^{n-1}+q-2\right) /\left(q^{n}-1\right) \leq 2 / q$. In all cases $|\theta(x)| \leq 2 \theta(1) / q \leq(3 N / q) \theta(1)-2 N$.

LEMMA 4.4. Let $\left(G, x, L_{J}\right)$ be an admissible triple with rank $(G) \stackrel{3}{\geq}$ 2. Let $\chi \in \operatorname{Irr}\left(P_{J}\right)$ and suppose that the irreducible constituents of $\chi_{U_{J}}$ are not $L_{J}^{\prime}$-invariant. Assume that $q \geq 3 N$ and that the Main Theorem holds for groups of smaller rank than $G$. Then $|\chi(x)| \leq$ $(3 N / q) \chi(1)-2 N+1$. 
Proof. By Clifford's Theorem, $\chi$ is induced from an inertia group $M U_{J}$, where $M \leq L_{J}$. By hypothesis, $L_{J}^{\prime} \nless M$. Mackey’s Theorem yields $\chi_{L_{J}}=\rho^{J}$, for some character $\rho$ of $M$. If $x$ is not conjugate in $L_{J}$ to an element of $M$, then $\chi(x)=0$. Hence we may assume $x \in$ $M$. Let $G_{0}=L_{J}^{\prime} M$. Then $\chi(x)=\chi_{L_{J}}(x)=\left(\rho^{G_{0}}\right)^{L_{J}}(x)=\sum \rho^{G_{0}}\left(x^{h}\right)$, where $h$ ranges over a transversal to $G_{0}$ in $L_{J}$.

It suffices to show that $\left|\rho^{G_{0}}\left(x^{h}\right)\right| \leq(3 N / q) \rho^{G_{0}}(1)-2 N+1$ for each $h$. A computation using the formula for induced characters shows that $\left|\rho^{G_{0}}\left(x^{h}\right) / \rho^{G_{0}}(1)\right| \leq 1_{M}^{G_{0}}\left(x^{h}\right) / 1_{M}^{G_{0}}(1)$. Thus if $1_{M}^{G_{0}}\left(x^{h}\right) \leq$ $(3 N / q) 1_{M}^{G_{0}}(1)-2 N+1$, then

$$
\begin{aligned}
\left|\rho^{G_{0}}\left(x^{h}\right) / \rho^{G_{0}}(1)\right| & \leq(3 N / q)+(1-2 N) / 1_{M}^{G_{0}}(1) \\
& \leq(3 N / q)+(1-2 N) / \rho^{G_{0}}(1)
\end{aligned}
$$

and so $\left|\rho^{G_{0}}\left(x^{h}\right)\right| \leq(3 N / q) \rho^{G_{0}}(1)-2 N+1$, the desired inequality. Hence we need only show that $1_{M}^{G_{0}}\left(x^{h}\right) \leq(3 N / q) 1_{M}^{G_{0}}(1)-2 N+1$. Since $L_{J}^{\prime} \nless M$, we have $M<G_{0}$.

Clearly $1_{G_{0}}$ has multiplicity 1 in $1_{M}^{G_{0}}$. If $\lambda$ is a linear constituent of $1_{M}^{G_{0}}$, then $\lambda_{M}=1_{M}$. Also $L_{J}^{\prime}=G_{0}^{\prime} \leq \operatorname{Ker} \lambda$. Hence $M L_{J}^{\prime}=G_{0} \leq$ $\operatorname{Ker} \lambda$, and so $\lambda=1_{G_{0}}$. Thus $1_{M}^{G_{0}}-1_{G_{0}}$ has no linear constituents.

Let $G_{1}=\left\langle x^{h}, L_{J}^{\prime}\right\rangle$. Then $\left(G_{1}, x^{h}\right)$ is an admissible pair by Lemma 1.6 , and $x^{h}$ centralizes no component of $G_{1}$. We have $G_{1} \leq G_{0}$ and $M G_{1}=G_{0}$. If $\alpha \neq 1_{G_{0}}$ is an irreducible constituent of $1_{M}^{G_{0}}$, then $\alpha$ is nonlinear by the preceding paragraph. Then $L_{J}^{\prime} \nless \operatorname{Ker} \alpha$, and so no irreducible constituent of $\alpha_{G_{1}}$ has $L_{J}^{\prime}$ in its kernel. Since $G_{1}^{\prime}=L_{J}^{\prime}$, all irreducible constituents of $\alpha_{G_{1}}$ are nonlinear. By Mackey's theorem, $1_{M \cap G_{1}}^{G_{1}}=\left(1_{M}^{G_{0}}\right)_{G_{1}}$. Hence $1_{M \cap G_{1}}^{G_{1}}-1_{G_{1}}=\left(1_{M}^{G_{0}}-1_{G_{0}}\right)_{G_{1}}$ is a sum of nonlinear irreducible constituents. Lemma 4.2 implies that $\left|\beta\left(x^{h}\right)\right| \leq(3 N / q) \beta(1)-N$ for every nonprincipal irreducible constituent $\beta$ of $1_{M \cap G_{1}}^{G_{1}}$. If $1_{M \cap G_{1}}^{G_{1}}-1_{G_{1}}$ is not irreducible, then $1_{M}^{G_{0}}\left(x^{h}\right)=$ $1_{M \cap G_{1}}^{G_{1}}\left(x^{h}\right)<1+(3 N / q) 1_{M \cap G_{1}}^{G_{1}}(1)-2 N=(3 N / q) 1_{M}^{G_{0}}(1)-2 N+1$, as desired.

Thus we may assume that $G_{1}$ is doubly transitive on the cosets of $M \cap G_{1}$. Let $1_{M \cap G_{1}}^{G_{1}}=1+\theta$, with $\theta \in \operatorname{Irr}\left(G_{1}\right)$. Then Lemma 4.3, applied to the admissible pair $\left(G_{1}, x^{h}\right)$, yields $1_{M}^{G_{0}}\left(x^{h}\right)=1_{M \cap G_{1}}^{G_{1}}\left(x^{h}\right) \leq$ $1+\left|\theta\left(x^{h}\right)\right| \leq 1+(3 N / q) \theta(1)-2 N<(3 N / q) 1_{M}^{G_{0}}(1)-2 N+1$, as desired. 
Proof of Main Theorem. We proceed by induction on the rank of $G$. If $G$ has rank 1 , then Lemma 4.1 yields $|\chi(x)| \leq 2 \chi(1) /(q-1)$. Hence $|\chi(x)| \leq 3 \chi(1) / q$. Thus it suffices to show that $3 \chi(1) / q \leq$ $(3 N / q) \chi(1)-N$. This is equivalent to $\chi(1) \geq q N /(3 N-3)$, which holds since $\chi(1) \geq(q-1) / 2$ and $q \geq 3 N \geq 15$.

Hence we assume that $\operatorname{rank}(G)>1$. If $x$ lies in no proper parabolic of $G$, then Theorem 1.11 says that $|\chi(x)| \leq 6 \chi(1) / q$, so we need to show that $6 \chi(1) / q \leq(3 N / q) \chi(1)-N$. This simplifies to $\chi(1) \geq q N /(3 N-6)$, which holds since $\chi(1) \geq\left(q^{2}-1\right) / 2$ by $[16$, p. 419]. We therefore assume that $x$ lies in a proper parabolic of $G$. Let $\left(G, x, L_{J}\right)$ be an admissible triple, where $x$ may be replaced by a $G$-conjugate. Write $\chi_{P_{J}}=\chi_{1}+\chi_{2}+\chi_{3}+\chi_{4}$, as in the introduction to this paper. By Theorems 2.10 and 2.17, Lemma 4.2, Theorem 3.5, Lemma 4.4 and our inductive hypothesis, $\left|\chi_{1}(x)\right| \leq$ $N-1,\left|\chi_{2}(x)\right| \leq(3 N / q) \chi_{2}(1)-N,\left|\chi_{3}(x)\right| \leq \chi_{3}(1) / q$, and $\left|\chi_{4}(x)\right| \leq$ $(3 N / q) \chi_{4}(1)-2 N+1$.

Let $\theta$ be an irreducible constituent of $\left(\chi_{3}\right)_{U_{J}}$. Then $U_{J} / \operatorname{Ker} \theta$ is extraspecial by Proposition 3.4 and its commutator factor group has order at least $q^{2}$ by the proof of Theorem 3.5. Hence $\theta(1) \geq q$, and so $\chi_{3}(1) \geq q$. It follows that $\chi_{3}(1) / q \leq(3 N / q) \chi_{3}(1)-N$, and so $\left|\chi_{3}(x)\right| \leq(3 N / q) \chi_{3}(1)-N$.

If $\chi_{4} \neq 0$, the triangle inequality shows that $|\chi(x)| \leq(3 N / q) \chi(1)-$ $N$. We may therefore assume $\chi_{4}=0$. Then $\chi_{3} \neq 0$, since otherwise $U_{J} \leq \operatorname{Ker} \chi$, which is impossible. If both $\chi_{2}$ and $\chi_{3}$ are nonzero, the triangle inequality and the conclusion of the preceding paragraph yield the desired bound for $|\chi(x)|$, so we assume that $\chi_{2}=\chi_{4}=0$.

Then $|\chi(x)| \leq N-1+\left(\chi_{3}(1) / q\right)$. It suffices to show that $N-$ $1+\left(\chi_{3}(1) / q\right) \leq(3 N / q) \chi_{3}(1)-N$. This is equivalent to $\chi_{3}(1) \geq$ $((2 N-1) /(3 N-1)) q$, which holds because $\chi_{3}(1) \geq q$.

COROLlaRy 4.5. Let $\bar{G}$ be a connected reductive algebraic group over $\overline{\mathrm{GF}(p)}$ whose commutator subgroup is simple, simply connected, and not special. Let $q$ and $N$ be as in the Main Theorem. Let $x$ be an admissible element of $\bar{G}_{\sigma}$. Let $G$ be a group satisfying $\left\langle\bar{G}_{\sigma}^{\prime}, x\right\rangle \leq G \leq$ $\bar{G}_{\sigma}$. Let $\chi \in \operatorname{Irr}(G)$ be nonlinear. Then $|\chi(x)| \leq(3 N / q) \chi(1)-N$.

Proof. Apply the Main Theorem to the constituents of the restriction of $\chi$ to $\left\langle\bar{G}_{\sigma}^{\prime}, x\right\rangle$. 


\section{REFERENCES}

[1] D. Alvis, Induce-restrict matrices for exceptional Weyl groups, preprint, 1981.

[2] H. Azad, M. Barry, and G. Seitz, On the structure of parabolic subgroups, Comm. Algebra, 18 (1990), 551-562.

[3] A. Borel and J. Tits, Eléments unipotents et sousgroupes paraboliques des groupes réductifs, Invent. Math., 12 (1971), 97-104.

[4] N. Bourbaki, Groupes et Algèbres de Lie, Ch. 4-6, Hermann, Paris, 1968.

[5] P. Cameron, Finite permutation groups and finite simple groups, Bull. London Math. Soc., 13 (1981), 1-22.

[6] R. Carter, Conjugacy classes in the Weyl group, Comp. Math., 25 (1972), 1-59.

[7] _ - Simple Groups of Lie Type, Wiley, London, 1972.

[8] __ Finite Groups of Lie Type: Conjugacy Classes and Irreducible Characters, Wiley, New York, 1985.

[9] C. Curtis, N. Iwahori, and R. Kilmoyer, Hecke algebras and characters of parabolic type of finite groups with BN-pairs, Publ. Math. IHES, 40 (1971), 81-116.

[10] C. Curtis and I. Reiner, Methods of Representation Theory, Volume 2, Wiley, New York, 1987.

[11] L. Dornhoff, Group Representation Theory (A), Dekker, New York, 1971.

[12] V. Ennola, On the characters of the finite unitary groups, Ann. Acad. Sc. Fenn., 323 (1963).

[13] R. Howlett and R. Kilmoyer, Principal series representations of finite groups with split BN-pairs, Comm. Algebra, 8 (1980), 543-583.

[14] B. Huppert, Endliche Gruppen, Springer, Berlin, 1967.

[15] I. M. Isaacs, Character Theory of Finite Groups, Academic Press, New York, 1976.

[16] V. Landazuri and G. Seitz, On the minimal degrees of projective representations of the finite Chevalley groups, J. Algebra, 32 (1974), 418-443.

[17] K. McGovern, Multiplicities of principal series representations of finite groups with split BN-pairs, J. Algebra, 77 (1982), 419-442.

[18] R. Steinberg, The representations of $\operatorname{GL}(3, q), \mathrm{GL}(4, q), \operatorname{PGL}(3, q)$, and PGL $(4, q)$, Canad. J. Math., 3 (1951), 225-235.

[19] J. Tits, Tabellen zu den einfachen Lie Gruppen und ihren Darstellungen, Lecture Notes in Mathematics, 40 (1967), Springer.

Received January 22, 1990 and in revised form July 17, 1990. Research partially supported by a grant from the National Security Agency and by a Wayne State University Career Development Chair.

WAyne State University

DETROIT, MI 48202 



\section{PACIFIC JOURNAL OF MATHEMATICS EDITORS}

\author{
V. S. VARADARAJAN \\ (Managing Editor) \\ University of California \\ Los Angeles, CA 90024-1555-05 \\ Herbert Clemens \\ University of Utah \\ Salt Lake City, UT 84112 \\ THOMAS ENRIGHT \\ University of California, San Diego \\ La Jolla, CA 92093
}

R. FINN

Stanford University

Stanford, CA 94305

HeRmann FlaschKa

University of Arizona

Tucson, AZ 85721

VAUGHAN F. R. JoNES

University of California

Berkeley, CA 94720

SteVen KeRCKHOFF

Stanford University

Stanford, CA 94305
C. C. MOORE

University of California

Berkeley, CA 94720

MaRTin ScharLemanN

University of California

Santa Barbara, CA 93106

HAROLd STARK

University of California, San Diego

La Jolla, CA 92093

\section{ASSOCIATE EDITORS \\ R. ARENS \\ E. F. BECKENBACH (1906-1982) \\ B. H. NeumanN \\ F. WoLf \\ (1904-1989) \\ K. YoshidA \\ SUPPORTING INSTITUTIONS \\ UNIVERSITY OF ARIZONA \\ UNIVERSITY OF BRITISH COLUMBIA \\ CALIFORNIA INSTITUTE OF TECHNOLOGY \\ UNIVERSITY OF CALIFORNIA \\ MONTANA STATE UNIVERSITY \\ UNIVERSITY OF NEVADA, RENO \\ NEW MEXICO STATE UNIVERSITY OREGON STATE UNIVERSITY \\ UNIVERSITY OF OREGON \\ UNIVERSITY OF SOUTHERN CALIFORNIA \\ STANFORD UNIVERSITY \\ UNIVERSITY OF HAWAII \\ UNIVERSITY OF TOKYO \\ UNIVERSITY OF UTAH \\ WASHINGTON STATE UNIVERSITY UNIVERSITY OF WASHINGTON}

The Supporting Institutions listed above contribute to the cost of publication of this Journal, but they are not owners or publishers and have no responsibility for its content or policies.

Mathematical papers intended for publication in the Pacific Journal of Mathematics should be in typed form or offset-reproduced (not dittoed), double spaced with large margins. Please do not use built up fractions in the text of the manuscript. However, you may use them in the displayed equations. Underline Greek letters in red, German in green, and script in blue. The first paragraph must be capable of being used separately as a synopsis of the entire paper. In particular it should contain no bibliographic references. Please propose a heading for the odd numbered pages of less than 35 characters. Manuscripts, in triplicate, may be sent to any one of the editors. Please classify according to the 1991 Mathematics Subject Classification scheme which can be found in the December index volumes of Mathematical Reviews. Supply name and address of author to whom proofs should be sent. All other communications should be addressed to the managing editor, or Elaine Barth, University of California, Los Angeles, California 90024-1555-05.

There are page-charges associated with articles appearing in the Pacific Journal of Mathematics. These charges are expected to be paid by the author's University, Government Agency or Company. If the author or authors do not have access to such Institutional support these charges are waived. Single authors will receive 50 free reprints; joint authors will receive a total of 100 free reprints. Additional copies may be obtained at cost in multiples of 50 .

The Pacific Journal of Mathematics (ISSN 0030-8730) is published monthly except for July and August. Regular subscription rate: $\$ 190.00$ a year (10 issues). Special rate: $\$ 95.00$ a year to individual members of supporting institutions.

Subscriptions, orders for numbers issued in the last three calendar years, and changes of address should be sent to Pacific Journal of Mathematics, P.O. Box 969, Carmel Valley, CA 93924, U.S.A. Old back numbers obtainable from Kraus Periodicals Co., Route 100, Millwood, NY 10546.

The Pacific Journal of Mathematics at P.O. Box 969, Carmel Valley, CA 93924 (ISSN 0030-8730) is published monthly except for July and August. Second-class postage paid at Carmel Valley, California 93924, and additional mailing offices. Postmaster: send address changes to Pacific Journal of Mathematics, P.O. Box 969, Carmel Valley, CA 93924.

PUBLISHED BY PACIFIC JOURNAL OF MATHEMATICS, A NON-PROFIT CORPORATION Copyright (C) 1991 by Pacific Journal of Mathematics 


\section{Pacific Journal of Mathematics}

Vol. 150, No. $2 \quad$ October, 1991

Selman Akbulut and Henry Churchill King, Rational structures on

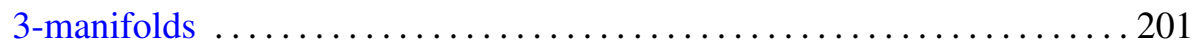

Mark Baker, On coverings of figure eight knot surgeries $\ldots \ldots \ldots \ldots \ldots 215$

Christopher Michael Brislawn, Traceable integral kernels on countably generated measure spaces . ...........................229

William Chin, Crossed products and generalized inner actions of Hopf algebras

Tadeusz Figiel, William Buhmann Johnson and Gideon Schechtman, Factorizations of natural embeddings of $l_{p}^{n}$ into $L_{r}$. II $\ldots \ldots \ldots \ldots \ldots 261$

David Howard Gluck, Character value estimates for groups of Lie type ... 279

Charn-Huen Kan, Norming vectors of linear operators between $L_{p}$

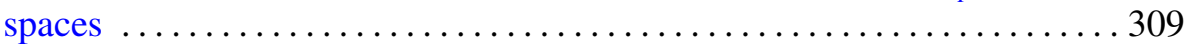

Marko Kranjc, Embedding a 2-complex $K$ in $\mathbb{R}^{4}$ when $H^{2}(K)$ is a cyclic

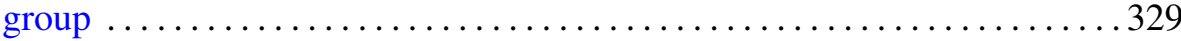

Ka-Lam Kueh, The remainder terms aspect of the theory of the Riemann

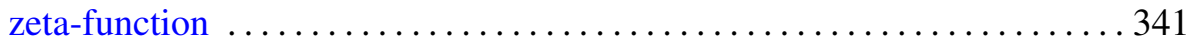

J. A. Marti, Sur la rigidité comparée de fonctions, distributions, ou hyperfonctions analytiques par rapport à un groupe de variables

Margherita Roggero and Paolo Valabrega, Chern classes and cohomology for rank 2 reflexive sheaves on $\mathbf{P}^{3}$ 